\section{Health-related and socio-demographic factors associated with frailty in the elderly: a systematic literature review}

\author{
Fatores sociodemográficos e de saúde associados \\ à fragilidade em idosos: uma revisão sistemática \\ de literatura
}

\author{
Factores sociodemográficos y de salud asociados \\ con la fragilidad en ancianos: una revisión \\ sistemática de la literatura
}

\begin{abstract}
${ }^{1}$ Escola Nacional de Saúde Pública Sergio Arouca, Fundação Oswaldo Cruz, Rio de Janeiro, Brasil.

2 Universidade Estadual de

Campinas, Campinas, Brasil.

Correspondence

A. C. Mello

Departamento de Ciências

Sociais, Escola Nacional de

Saúde Pública Sergio Arouca, Fundação Oswaldo Cruz.

Rua Leopoldo Bulhões 1480

Rio de Janeiro, RJ 21041-210,

Brasil.

amandita.nut@gmail.com
\end{abstract}

\begin{abstract}
Frailty is a syndrome that leads to practical harm in the lives of elders, since it is related to increased risk of dependency, falls, hospitalization, institutionalization, and death. The objective of this systematic review was to identify the socio-demographic, psycho-behavioral, healthrelated, nutritional, and lifestyle factors associated with frailty in the elderly. A total of 4,183 studies published from 2001 to 2013 were detected in the databases, and 182 complete articles were selected. After a comprehensive reading and application of selection criteria, 35 eligible articles remained for analysis. The main factors associated with frailty were: age, female gender, black race/color, schooling, income, cardiovascular diseases, number of comorbidities/diseases, functional incapacity, poor self-rated health, depressive symptoms, cognitive function, body mass index, smoking, and alcohol use. Knowledge of the complexity of determinants of frailty can assist the formulation of measures for prevention and early intervention, thereby contributing to better quality of life for the elderly.
\end{abstract}

Frail Elderly; Quality of Life; Risk Factors
Amanda de Carvalho Mello 1 Elyne Montenegro Engstrom 1 Luciana Correia Alves ${ }^{2}$

\section{Resumo}

A fragilidade é uma síndrome que gera prejuízos práticos à vida do idoso, pois está relacionada à maior risco de dependência, quedas, hospitalização, institucionalização e morte. O objetivo desta revisão sistemática foi identificar os fatores sociodemográficos, psicocomportamentais, de condições de saúde, estado nutricional e estilo de vida associados à fragilidade em idosos. Quatro mil cento e oitenta e três trabalhos publicados entre 2001 e 2013 foram detectados nas bases bibliográficas e selecionados 182 artigos completos. Após a leitura integral e aplicação dos critérios de seleção, restaram 35 artigos elegíveis para análise. Os principais fatores associados foram: idade, sexo feminino, raça/cor da pele preta, escolaridade, renda, doenças cardiovasculares, número de comorbidades/doenças, incapacidade funcional, autoavaliação de saúde ruim, sintomas depressivos, função cognitiva, índice de massa corporal, tabagismo e uso de álcool. O conhecimento da complexidade dos determinantes da fragilidade auxilia na formulação de ações de prevenção e intervenção precoce, garantindo maior qualidade de vida.

Idoso Fragilizado; Qualidade de Vida; Fatores de Risco 


\section{Introduction}

Frailty in the elderly is defined as a clinical syndrome characterized by a decrease in energy reserve, strength, and performance, resulting in a cumulative decline in multiple physiological systems, leading to a state of greater vulnerability 1,2 . This condition causes practical harm to the life of elders and their families, both clinically and psychosocially, since it is associated with greater risk of adverse consequences such as dependency, falls, hospitalization, institutionalization, and death 1,2,3,4,5. Prevalence in Americans is $6.3 \%$ 2, and in Brazilians it varies from 10 to $25 \%$ in persons above 65 years and $46 \%$ above 85 years 4 .

The syndrome should not be confused with functional dependency or comorbidity. Frailty refers to the fact that a person needs help or requires assistance to perform an activity, or fails to perform it. Individuals are classified as dependent when they need help from someone else or are unable to perform a task 3,6. Meanwhile, comorbidity is a general concept that encompasses the presence of several diagnosed illnesses 3 .

Studies have focused on understanding the causes and pathophysiology of frailty, defining and improving criteria to identify elderly at risk and analyzing factors that influence development of the syndrome. There are different definitions for the identification of frailty. The most widely used is that of Fried et al. 2, who define frail elderly as those with three or more of the following indicators: unintentional weight loss, low level of physical activity, reduced grip strength, reduced gait speed, and self-reported fatigue. Another criterion that has been discussed in the scientific literature is that of Rockwood et al. 7 , which adds cognitive and emotional aspects to the diagnostic indicators. There are still other criteria, with no consensus in the academic community on the best approach to diagnosis; however, in a recently published report 1 , experts agreed that health professionals should choose a well-validated model among the existing ones.

Since the factors related to the syndrome are not fully known, it is extremely important to understand it in order for targeted measures to be planned and implemented. Many of these health problems can be prevented at the primary care level, as long as healthcare professionals are alert to the determinant factors for the syndrome and aware of the importance of early detection. Studies have shown that various physiological, sociodemographic, psychological, and nutritional factors may be involved in the origin of frailty, in addition to related comorbidities 5,8,9.
The current systematic review aims to identify socio-demographic, psycho-behavioral, health-related, nutritional, and lifestyle factors associated with frailty in the elderly.

\section{Methods \\ Databases and search strategy}

Articles published from 2001 to 2013 were selected from the following databases: MEDLINE via PubMed, Scopus, LILACS, and ISI Web of Knowledge. The descriptors and MesH terms consulted in the search engines were: "age factors" OR "risk factors" OR "socioeconomic factors" OR "demographic factors" OR "clinical factors", "biological factors" OR "behavior factors" OR “elderly nutrition” OR "nutrition”, "health status" OR "epidemiological factors" OR "elder nutritional physiological phenomena" in the field all words in the literature bases, in combination using the Boolean connector $A N D$ with "aging” OR “aged" OR "elderly" OR "senescence", in the field all words and associated by the Boolean connector $A N D$ with "frail elderly" OR "frailty" OR "fragility" OR "elderly frail" OR "frail older adults" in the field Title and/or abstract + key words. Articles in English, Spanish, and Portuguese were selected.

\section{Selection criteria}

The review used the following selection criteria: original scientific articles published in Brazilian or international periodicals; publication from 2001 to March 2013; study population 60 years or older; observational study design (cross-sectional, cohort, or case-control); individual selection by probabilistic sample or article showing the sampling design; and identification of factors associated with frailty in the elderly as the principal or secondary objective.

Importantly, there are different diagnostic criteria for frailty, with no consensus in the literature as to the most adequate markers for its identification. However a widely used and wellaccepted criterion in the scientific community is that of Fried et al. 2, published in 2001. Based on a study of Americans participating in the Cardiovascular Health Study, the group proposed that the syndrome's pathophysiology can be identified by a phenotype, using five measurable components:

- Self-reported unintentional weight loss of $4.5 \mathrm{~kg}$ or $5 \%$ of body weight in the previous year;

- Self-reported fatigue assessed by the following: "I felt tired all the time" and "I could not get 
going”, from the depression scale of the Center for Epidemiological Studies (CES-D) 10;

- Decreased grip strength, measured with a dynamometer in the dominant hand, stratified by gender and body mass index (BMI) quartiles;

- Low level of physical activity measured as weekly energy expenditure in kcal, with information obtained from the reduced version of the Minnesota Leisure Time Activity Questionnaire 11, stratified by gender;

- Decreased gait speed in seconds, calculated by recording the time to walk $4.6 \mathrm{~m}$ at a comfortable pace, stratified by gender and mean height.

The presence of three or more components defines a frail elder. The presence of one to two identifies those at high risk of developing the syndrome (pre-frail). We only selected articles that reported using this criterion to identify frailty, so the article search began in 2001, the year this definition was published.

\section{Data extraction}

Article selection and data extraction were performed independently by three reviewers, using a standardized instrument containing: country and study site; sampling number; type of sample; study design; characteristics of sample member; study variables; criterion used to identify frailty; statistical technique; principal results; and limitations.

\section{Assessment of risk of bias}

Assessment of the articles included in the analysis used verification of the risk of bias, as suggested by the Cochrane Collaboration ${ }^{12}$. To orient the assessment of this risk, an adapted version of the Newcastle-Ottawa Scale 13 was used (Table 1). The original Newcastle-Ottawa Scale was developed to assess the quality of observational studies and contains eight items that analyze three dimensions: selection, comparability, and outcome (in the case of cohort studies) or exposure (case-control). For each item there is a series of options in which the one that best reflects quality is marked with a star; the more the stars, the higher the study's quality 14 . In the current study, questions were adjusted to investigate exposure and outcome (frailty according to the definition

Table 1

Adaptation of the Newcastle-Ottawa Scale 13 to assess quality of studies using the definition of frailty according to Fried et

al. 2 as the outcome variable.

Exposure

Independent variables

a) Secure recording + primary measurements * (low risk of bias)

b) Structured interview + primary measurements, without knowledge of

outcome * (low risk of bias)

c) Interview with knowledge of outcome (high risk of bias)

d) Non-secures sources and self-assessment (high risk of bias)

e) Does not describe clearly (uncertain risk of bias)

\section{Outcome}

Is the assessment of frailty

adequate?

Representativeness of sample

Selection of participants

Definition of the control group or cohort (only for longitudinal studies)

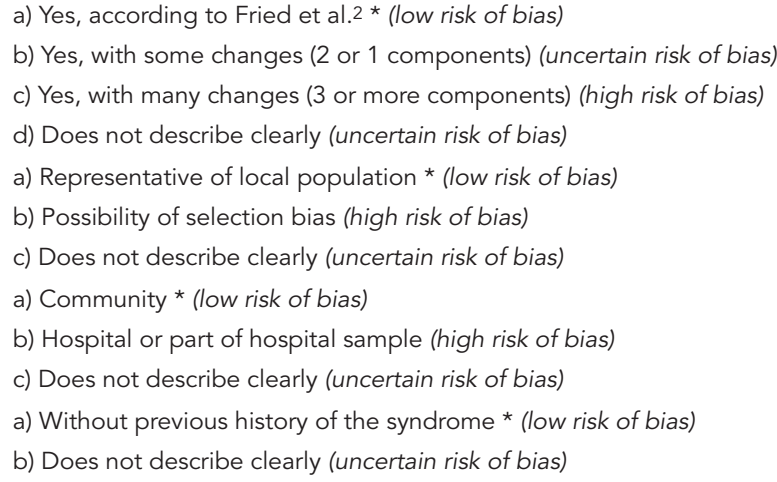

* Represents an item for classification of low risk of bias. 
by Fried et al. 2), and risk of bias was divided into low risk of bias, uncertain risk of bias, and high risk of bias, according to the item being assessed. Each star represents an item for classification of low risk of bias.

\section{Results}

The literature search identified 4,183 publications. Of these, we eliminated 629 duplicates that came from two or more databases, and after reading the titles and abstracts, 3,372 were ruled out because frailty was an independent variable, the study was designed as an intervention, review, or validation of a diagnostic criterion, or the publication was a book or journal commentary or editorial. A total of 182 complete articles were selected for analysis. After reading them and applying the selection criteria, 35 eligible articles were left. Figure 1 shows the study selection flow.

\section{Overall study characteristics}

In the 35 studies, the data collection period ranged from 1989 to 2011 . The year with the most publications was $2012(\mathrm{n}=10)$. Most studies were on North American participants $(\mathrm{n}=12)$, followed by Europeans $(\mathrm{n}=11)$, Latin Americans $(n=9)$, and Asians $(n=3)$. The number of subjects varied from 77 to 40,657 , and most studies had samples greater than 600 individuals. Age of

Figure 1

Flow chart for selection of articles for analysis.

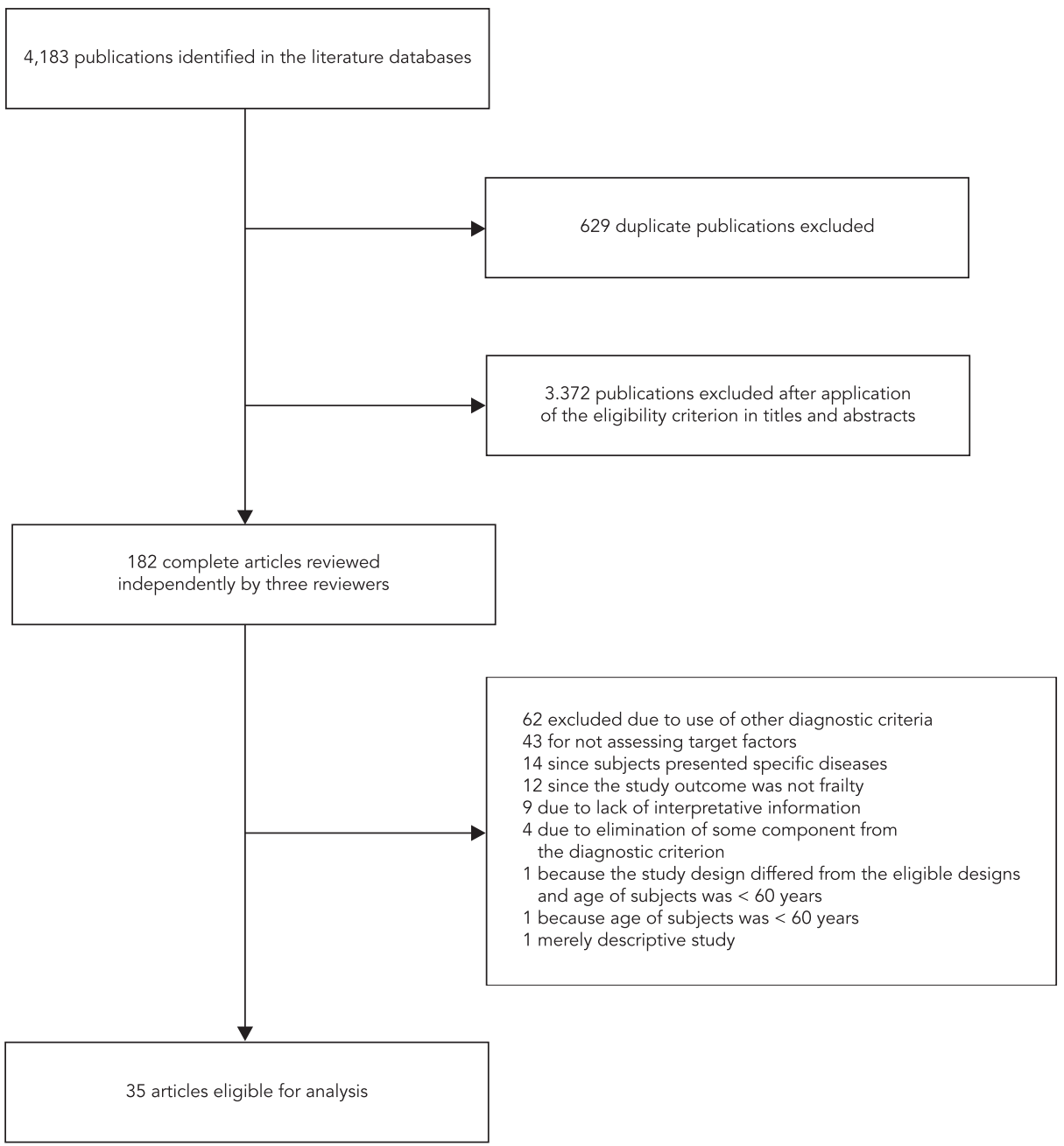


the elderly was greater than 65 years, except for three studies, in which it was greater than 60 . The results analyzed in this article are mainly from cross-sectional studies $(\mathrm{n}=27)$.

Table 2 shows details on the main characteristics of the 35 studies, with the design, independent variables, statistical technique, principal results, and limitations listed by authors.

The most widely studied independent variables were demographic $(n=33)$, diseases and health conditions $(n=30)$, socioeconomic $(n=$ $30)$, psycho-behavioral $(\mathrm{n}=23)$, and nutritional $(n=17)$, and the least studied were lifestyle variables $(n=11)$. The majority of the studies used logistic regression models $(n=24)$. All the results presented here were statistically significant.

\section{Demographic and socioeconomic factors and frailty}

Of the 35 studies, demographic factors were assessed by 33 and socioeconomic factors by 30 . The most frequently assessed demographic variable was age $(\mathrm{n}=31)$ and the most common socioeconomic value was schooling or educational level $(n=27)$. In general, age, black race/color, and female gender showed a positive association with frailty, while there was an inverse association between frailty and schooling and income.

\section{Diseases, health conditions, and frailty}

The principal diseases assessed by the studies were cardiovascular diseases (CVD) $(n=17)$, diabetes mellitus $(n=17)$, systemic arterial hypertension $(\mathrm{SAH})(\mathrm{n}=14)$, pulmonary diseases $(\mathrm{n}=10)$, arthritis $(\mathrm{n}=11)$, cancer $(\mathrm{n}=8)$, and stroke $(n=7)$. Fourteen studies also included comorbidities/diseases as an independent variable. Frailty showed a positive association especially with CVD and number of comorbidities/ diseases. As predicted, no disease showed an inverse association with frailty or was considered to have a protective effect.

Sixteen studies investigated functional status, measured mainly by activities of daily living (ADL) and instrumental activities of daily living (IADL), and diagnosis of functional incapacity showed a positive association with frailty in nine.

Eight studies analyzed self-rated health and found a positive association between poor selfrated health and frailty.

\section{Psycho-behavioral factors and frailty}

Depressive symptoms were assessed in 17 studies, and cognitive function was tested in 15 . The instrument most widely used to assess cognitive function was the Mini Mental State Examination (MMSE) 15, and the elderly that tested highest were considered to have the best cognitive function. An inverse association was found between cognitive function and frailty, while depressive symptoms showed a positive association with the syndrome.

\section{Nutritional status, lifestyle and frailty}

The most widely assessed nutritional variable was BMI $(n=13)$. Lifestyle factors were the least analyzed in the selected articles and included smoking ( $\mathrm{n}=10)$, alcohol consumption $(\mathrm{n}=6)$, and physical activity $(n=2)$. A positive association was found between smoking and frailty in two studies, and an inverse association was observed between alcohol and frailty in three studies. Most of the studies found a positive association between frailty and BMI, and two studies showed that underweight elderly according to BMI had a positive association with frailty.

\section{Limitations listed by the authors of the articles analyzed in the final sample}

The limitations most frequently cited by the authors of the selected articles were: cross-sectional design (not allowing causal inferences); adaptations of scales suggested by Fried et al. 2; and selfreporting of data.

\section{Assessment of risk of bias}

Table 3 summarizes the assessment of risk of bias in the studies, and Figure 2 shows the graph for each question in the adapted Newcastle-Ottawa Scale 13 .

In relation to analysis of the risk of bias, 34 studies collected information on the independent variables using a structured interview, anthropometric measurements, and clinical tests (low risk of bias), and only one failed to clearly describe the method (uncertain risk of bias).

As for participant selection, all were local community-dwelling, non-institutionalized elders (low risk of bias).

More than half of the studies $(n=19)$ showed changes in three or more of the components in the criterion adopted by Fried et al. 2 (high risk of bias).

Only one article mentioned that the sample was representative of the local population (low risk of bias).

Only one of the longitudinal studies $(n=7)$ specified that the sample did not present the syndrome at the beginning of the cohort (low risk of bias). 
Table 2

Factors associated with frailty in the elderly according to observational studies.

\begin{tabular}{|c|c|c|c|c|c|c|c|}
\hline \multirow[t]{2}{*}{ Article (year) } & \multirow[t]{2}{*}{ Study design } & \multirow{2}{*}{$\begin{array}{l}\text { Independent } \\
\text { variables }\end{array}$} & \multirow{2}{*}{$\begin{array}{l}\text { Statistical } \\
\text { technique }\end{array}$} & \multirow{2}{*}{$\begin{array}{l}\text { Variables used } \\
\text { in adjustment } \\
\text { of final model }\end{array}$} & \multicolumn{2}{|c|}{ Principal results } & \multirow{2}{*}{$\begin{array}{l}\text { Methodological } \\
\text { limitations } \\
\text { according to the } \\
\text { authors }\end{array}$} \\
\hline & & & & & $\begin{array}{l}\text { Positive } \\
\text { association } \\
\text { with frailty }\end{array}$ & $\begin{array}{c}\text { Inverse } \\
\text { association } \\
\text { with frailty }\end{array}$ & \\
\hline $\begin{array}{l}\text { Fried et al. } 2 \\
\text { (2001) }\end{array}$ & Longitudinal & $\begin{array}{c}\text { Demographic: } \\
\text { age, gender, race } \\
\text { Socioeconomic: } \\
\text { schooling, } \\
\text { income, living } \\
\text { alone } \\
\text { Diseases and } \\
\text { health conditions: } \\
\text { number of } \\
\text { diseases, } \\
\text { comorbidities } \\
\text { (CVD, COPD, } \\
\text { SAH, diabetes } \\
\text { mellitus, arthritis, } \\
\text { cancer) } \\
\text { Psycho- } \\
\text { behavioral } \\
\text { cognitive } \\
\text { function, } \\
\text { depressive } \\
\text { symptoms } \\
\text { Other: ADL, } \\
\text { IADL, self-rated } \\
\text { health, functional } \\
\text { incapacity }\end{array}$ & $\begin{array}{c}\text { Cochran-Mantel- } \\
\text { Haenszel test }\end{array}$ & Age & $\begin{array}{c}\text { Female gender, } \\
\text { black race, } \\
\text { poor self-rated } \\
\text { health, living } \\
\text { alone, number } \\
\text { of diseases (2 or } \\
\text { more), CVD, lung } \\
\text { diseases, arthritis } \\
\text { and diabetes, } \\
\text { functional } \\
\text { incapacity and } \\
\text { depressive } \\
\text { symptoms }\end{array}$ & $\begin{array}{c}\text { Income, } \\
\text { schooling and } \\
\text { cognitive function }\end{array}$ & $\begin{array}{l}\text { Measures used in } \\
\text { operationalization } \\
\text { of the frailty } \\
\text { criterion were } \\
\text { limited to those } \\
\text { used during the } \\
\text { data collection } \\
\text { for other study } \\
\text { purposes; weight } \\
\text { at the beginning } \\
\text { of study was self- } \\
\text { reported }\end{array}$ \\
\hline $\begin{array}{l}\text { Newman et } \\
\text { al. } 27 \text { (2001) }\end{array}$ & Longitudinal & $\begin{array}{l}\text { Demographic: } \\
\text { age, gender, race } \\
\text { Socioeconomic: } \\
\text { schooling, } \\
\text { income } \\
\text { Diseases and } \\
\text { health conditions: } \\
\text { CVD } \\
\text { Psycho- } \\
\text { behavioral: } \\
\text { cognitive } \\
\text { function, } \\
\text { depressive } \\
\text { symptoms } \\
\text { Other: ADL, } \\
\text { IADL, self-rated } \\
\text { health, functional } \\
\text { incapacity }\end{array}$ & $\begin{array}{c}\text { Bivariate analysis } \\
\text { and multinomial } \\
\text { logistic } \\
\text { regression }\end{array}$ & $\begin{array}{l}\text { Age, gender } \\
\text { and race }\end{array}$ & $\begin{array}{l}\text { Female gender, } \\
\text { black race, CVD }\end{array}$ & $\begin{array}{c}\text { Income, } \\
\text { schooling and } \\
\text { cognitive function }\end{array}$ & $\begin{array}{c}\text { Use of self- } \\
\text { reported } \\
\text { measures; few } \\
\text { details on fatigue } \\
\text { and energy } \\
\text { expenditure (Kcal); } \\
\text { cross-sectional } \\
\text { study, does not } \\
\text { allow causal } \\
\text { inferences }\end{array}$ \\
\hline
\end{tabular}

(continues) 
Table 2 (continued)

\begin{tabular}{|c|c|c|c|c|c|c|c|}
\hline \multirow[t]{2}{*}{ Article (year) } & \multirow[t]{2}{*}{ Study design } & \multirow{2}{*}{$\begin{array}{l}\text { Independent } \\
\text { variables }\end{array}$} & \multirow{2}{*}{$\begin{array}{l}\text { Statistical } \\
\text { technique }\end{array}$} & \multirow{2}{*}{$\begin{array}{l}\text { Variables used } \\
\text { in adjustment } \\
\text { of final model }\end{array}$} & \multicolumn{2}{|c|}{ Principal results } & \multirow{2}{*}{$\begin{array}{l}\text { Methodological } \\
\text { limitations } \\
\text { according to the } \\
\text { authors }\end{array}$} \\
\hline & & & & & $\begin{array}{l}\text { Positive } \\
\text { association } \\
\text { with frailty }\end{array}$ & $\begin{array}{c}\text { Inverse } \\
\text { association } \\
\text { with frailty }\end{array}$ & \\
\hline $\begin{array}{l}\text { Blaum et al. } 33 \\
\text { (2005) }\end{array}$ & Cross-sectional & $\begin{array}{l}\text { Demographic: age, } \\
\text { gender, race } \\
\text { Socioeconomic: } \\
\text { schooling } \\
\text { Diseases and } \\
\text { health conditions: } \\
\text { CVD, COPD, } \\
\text { osteoarthritis } \\
\text { Nutritional status: } \\
\text { BMI } \\
\text { Other: functional } \\
\text { status (ADL and } \\
\text { IADL) }\end{array}$ & $\begin{array}{l}\text { Multinomial } \\
\text { logistic } \\
\text { regression }\end{array}$ & $\begin{array}{l}\text { All variables } \\
\text { listed }\end{array}$ & $\begin{array}{l}\text { Pre-frailty } \\
\text { and frailty: } \\
\text { overweight/ } \\
\text { obesity }\end{array}$ & - & - \\
\hline $\begin{array}{l}\text { Woods et al. } \\
29 \text { (2005) }\end{array}$ & Longitudinal & $\begin{array}{c}\text { Demographic: age, } \\
\text { race } \\
\text { Socioeconomic: } \\
\text { schooling, income, } \\
\text { living alone } \\
\text { Diseases and } \\
\text { health conditions: } \\
\text { comorbidity (CVD, } \\
\text { SAH, diabetes } \\
\text { mellitus, fractures, } \\
\text { COPD, arthritis, } \\
\text { stroke) } \\
\text { Lifestyle: smoking, } \\
\text { alcohol use } \\
\text { Nutritional status: } \\
\text { BMI } \\
\text { Psycho-behavioral: } \\
\text { depressive } \\
\text { symptoms } \\
\text { Other: functional } \\
\text { status (ADL) }\end{array}$ & $\begin{array}{c}\text { Bivariate analysis } \\
\text { and multivariate } \\
\text { logistic } \\
\text { regression }\end{array}$ & All & $\begin{array}{l}\text { Age, black and } \\
\text { Hispanic race, } \\
\text { underweight } \\
\text { and overweight/ } \\
\text { obesity (BMI), } \\
\text { comorbidities, } \\
\text { depressive } \\
\text { symptoms, } \\
\text { history of fall, } \\
\text { functional } \\
\text { incapacity, poor } \\
\text { self-rated health, } \\
\text { smoking }\end{array}$ & $\begin{array}{c}\text { Income, } \\
\text { schooling, living } \\
\text { alone, alcohol } \\
\text { use }\end{array}$ & $\begin{array}{l}\text { Lack of } \\
\text { information on } \\
\text { physical activity } \\
\text { and unintentional } \\
\text { weight loss; study } \\
\text { limited to sample } \\
\text { of women, not } \\
\text { possible to make } \\
\text { inferences for } \\
\text { men; low cognitive } \\
\text { function may be } \\
\text { confounder }\end{array}$ \\
\hline $\begin{array}{l}\text { Hirsch et al. } 25 \\
\text { (2006) }\end{array}$ & Cross-sectional & $\begin{array}{l}\text { Demographic: age, } \\
\text { gender, race } \\
\text { Socioeconomic: } \\
\text { schooling, income } \\
\text { Diseases and } \\
\text { health conditions: } \\
\text { CVD, SAH, COPD, } \\
\text { diabetes mellitus } \\
\text { and arthritis } \\
\text { Psycho-behavioral: } \\
\text { cognitive function } \\
\text { Nutritional status: } \\
\text { BMI }\end{array}$ & $\begin{array}{l}\text { Multinomial } \\
\text { logistic } \\
\text { regression }\end{array}$ & $\begin{array}{l}\text { Gender and } \\
\text { obesity }\end{array}$ & $\begin{array}{l}\text { Non-obese } \\
\text { blacks }\end{array}$ & - & $\begin{array}{l}\text { Cross-sectional } \\
\text { study, does not } \\
\text { allow causal } \\
\text { inferences; } \\
\text { possible selection } \\
\text { bias in subject } \\
\text { recruitment; } \\
\text { introduction of } \\
\text { bias by excluding } \\
\text { individuals with } \\
\text { missing data }\end{array}$ \\
\hline
\end{tabular}

(continues) 
Table 2 (continued)

\begin{tabular}{|c|c|c|c|c|c|c|c|}
\hline \multirow[t]{2}{*}{ Article (year) } & \multirow[t]{2}{*}{ Study design } & \multirow{2}{*}{$\begin{array}{l}\text { Independent } \\
\text { variables }\end{array}$} & \multirow{2}{*}{$\begin{array}{l}\text { Statistical } \\
\text { technique }\end{array}$} & \multirow{2}{*}{$\begin{array}{l}\text { Variables used } \\
\text { in adjustment } \\
\text { of final model }\end{array}$} & \multicolumn{2}{|c|}{ Principal results } & \multirow{2}{*}{$\begin{array}{l}\text { Methodological } \\
\text { limitations } \\
\text { according to the } \\
\text { authors }\end{array}$} \\
\hline & & & & & $\begin{array}{l}\text { Positive } \\
\text { association } \\
\text { with frailty }\end{array}$ & $\begin{array}{l}\text { Inverse } \\
\text { association } \\
\text { with frailty }\end{array}$ & \\
\hline $\begin{array}{l}\text { Michelon et } \\
\text { al. } 17 \text { (2006) }\end{array}$ & Cross-sectional & $\begin{array}{l}\text { Demographic: age, } \\
\text { race, marital status } \\
\text { Socioeconomic: } \\
\text { schooling, income } \\
\text { Lifestyle: smoking, } \\
\text { alcohol use } \\
\text { Nutritional status: } \\
\text { BMI, self-reported } \\
\text { weight loss and } \\
\text { low appetite } \\
\text { Other: functional } \\
\text { incapacity, self- } \\
\text { rated health }\end{array}$ & Bivariate analysis & - & $\begin{array}{l}\text { Age, black race, } \\
\text { widowhood, } \\
\text { smoking, } \\
\text { self-reported } \\
\text { weight loss, low } \\
\text { appetite, use } \\
\text { of dentures, } \\
\text { problems with } \\
\text { swallowing, poor } \\
\text { self-rated health, } \\
\text { BMI, functional } \\
\text { incapacity }\end{array}$ & $\begin{array}{l}\text { Income, } \\
\text { schooling, } \\
\text { alcohol use }\end{array}$ & $\begin{array}{l}\text { Cross-sectional } \\
\text { study, does not } \\
\text { allow causal } \\
\text { inferences; non- } \\
\text { inclusion of dietary } \\
\text { analyses }\end{array}$ \\
\hline $\begin{array}{l}\text { Semba et al. } 34 \\
(2006)\end{array}$ & Longitudinal & $\begin{array}{l}\text { Demographic: age, } \\
\text { race, marital status } \\
\text { Socioeconomic: } \\
\text { schooling, income } \\
\text { Lifestyle: smoking, } \\
\text { alcohol use } \\
\text { Nutritional status: } \\
\text { BMI, self-reported } \\
\text { weight loss and } \\
\text { low appetite } \\
\text { Other: functional } \\
\text { incapacity, self- } \\
\text { rated health }\end{array}$ & Bivariate analysis & - & $\begin{array}{c}\text { Age, CVD, low } \\
\text { appetite }\end{array}$ & BMI, schooling & - \\
\hline $\begin{array}{l}\text { Ávila-Funes et } \\
\text { al. } 16 \text { (2008) }\end{array}$ & Longitudinal & $\begin{array}{l}\text { Demographic: } \\
\text { age, gender, race, } \\
\text { marital status } \\
\text { Socioeconomic: } \\
\text { schooling, income, } \\
\text { living alone } \\
\text { Diseases and } \\
\text { health conditions: } \\
\text { CVD, COPD, } \\
\text { diabetes mellitus, } \\
\text { SAH, cancer and } \\
\text { arthrosis } \\
\text { Lifestyle: smoking, } \\
\text { alcohol use } \\
\text { Psycho-behavioral: } \\
\text { cognitive function } \\
\text { and depressive } \\
\text { symptoms } \\
\text { Nutritional status: } \\
\text { food intake } \\
\text { Other: functional } \\
\text { incapacity, self- } \\
\text { rated health }\end{array}$ & Bivariate analysis & - & $\begin{array}{l}\text { Age, female } \\
\text { gender, chronic } \\
\text { diseases, } \\
\text { functional } \\
\text { incapacity, } \\
\text { depressive } \\
\text { symptoms, poor } \\
\text { self-rated health }\end{array}$ & $\begin{array}{l}\text { Schooling, } \\
\text { income, cognitive } \\
\text { function, alcohol } \\
\text { use and smoking }\end{array}$ & $\begin{array}{l}\text { Adaptation of } \\
\text { scale from Fried } \\
\text { et al. } 2 \text { for weight } \\
\text { loss and grip } \\
\text { strength }\end{array}$ \\
\hline
\end{tabular}

(continues) 
Table 2 (continued)

\begin{tabular}{|c|c|c|c|c|c|c|c|}
\hline \multirow[t]{2}{*}{ Article (year) } & \multirow[t]{2}{*}{ Study design } & \multirow{2}{*}{$\begin{array}{c}\text { Independent } \\
\text { variables }\end{array}$} & \multirow{2}{*}{$\begin{array}{l}\text { Statistical } \\
\text { technique }\end{array}$} & \multirow{2}{*}{$\begin{array}{l}\text { Variables used } \\
\text { in adjustment } \\
\text { of final model }\end{array}$} & \multicolumn{2}{|c|}{ Principal results } & \multirow{2}{*}{$\begin{array}{l}\text { Methodological } \\
\text { limitations } \\
\text { according to the } \\
\text { authors }\end{array}$} \\
\hline & & & & & $\begin{array}{l}\text { Positive } \\
\text { association } \\
\text { with frailty }\end{array}$ & $\begin{array}{c}\text { Inverse } \\
\text { association } \\
\text { with frailty }\end{array}$ & \\
\hline $\begin{array}{l}\text { Alvarado et } \\
\text { al. } 39 \text { (2008) }\end{array}$ & Cross-sectional & $\begin{array}{l}\text { Demographic: } \\
\text { age, gender, } \\
\text { marital status } \\
\text { Socioeconomic: } \\
\text { schooling, } \\
\text { socioeconomic } \\
\text { status } \\
\text { Diseases and } \\
\text { health conditions: } \\
\text { comorbidities, } \\
\text { childhood and } \\
\text { adulthood } \\
\text { diseases } \\
\text { Lifestyle: physical } \\
\text { activity } \\
\text { Other: social } \\
\text { history }\end{array}$ & $\begin{array}{l}\text { Logistic } \\
\text { regression }\end{array}$ & $\begin{array}{l}\text { Social history, } \\
\text { comorbidity, } \\
\text { gender, } \\
\text { childhood } \\
\text { diseases, } \\
\text { schooling, } \\
\text { occupation, } \\
\text { marital status, } \\
\text { socioeconomic } \\
\text { status }\end{array}$ & Female gender & $\begin{array}{l}\text { Living conditions } \\
\text { in childhood }\end{array}$ & $\begin{array}{l}\text { Adaptation } \\
\text { of scale from } \\
\text { Fried et al. } 2 \text { for } \\
\text { weight and gait } \\
\text { speed; absence } \\
\text { of mortality } \\
\text { study; recall bias } \\
\text { for information } \\
\text { on poverty in } \\
\text { childhood }\end{array}$ \\
\hline $\begin{array}{l}\text { Chaves et } \\
\text { al. } 35 \text { (2008) }\end{array}$ & Cross-sectional & $\begin{array}{l}\text { Demographic: } \\
\text { age, race } \\
\text { Diseases and } \\
\text { health conditions: } \\
\text { CVD, diabetes } \\
\text { mellitus } \\
\text { Psycho- } \\
\text { behavioral: } \\
\text { cognitive } \\
\text { function, } \\
\text { depressive } \\
\text { symptoms } \\
\text { Other: self-rated } \\
\text { health }\end{array}$ & $\begin{array}{l}\text { Multivariate } \\
\text { logistic } \\
\text { regression }\end{array}$ & - & $\begin{array}{l}\text { Age, depressive } \\
\text { symptoms, } \mathrm{CHF}\end{array}$ & - & $\begin{array}{l}\text { Cross-sectional } \\
\text { study, does not } \\
\text { allow causal } \\
\text { inferences }\end{array}$ \\
\hline $\begin{array}{l}\text { Endeshaw et } \\
\text { al. } 40 \text { (2009) }\end{array}$ & Cross-sectional & $\begin{array}{l}\text { Demographic: } \\
\text { age, race } \\
\text { Diseases and } \\
\text { health conditions: } \\
\text { SAH, diabetes } \\
\text { mellitus, CVD, } \\
\text { stroke } \\
\text { Psycho- } \\
\text { behavioral: } \\
\text { cognitive function } \\
\text { Nutritional status: } \\
\text { BMI } \\
\text { Other: functional } \\
\text { status (ADL) }\end{array}$ & $\begin{array}{l}\text { Multivariate } \\
\text { logistic } \\
\text { regression }\end{array}$ & - & $\begin{array}{c}\text { In men: age, } \\
\text { black race, CVD, } \\
\text { stroke } \\
\text { In women: age, } \\
\text { obesity }\end{array}$ & - & $\begin{array}{l}\text { Cross-sectional } \\
\text { study, does not } \\
\text { allow causal } \\
\text { inferences }\end{array}$ \\
\hline
\end{tabular}

(continues) 
Table 2 (continued)

\begin{tabular}{|c|c|c|c|c|c|c|c|}
\hline \multirow[t]{2}{*}{ Article (year) } & \multirow[t]{2}{*}{ Study design } & \multirow{2}{*}{$\begin{array}{l}\text { Independent } \\
\text { variables }\end{array}$} & \multirow{2}{*}{$\begin{array}{l}\text { Statistical } \\
\text { technique }\end{array}$} & \multirow{2}{*}{$\begin{array}{l}\text { Variables used } \\
\text { in adjustment } \\
\text { of final model }\end{array}$} & \multicolumn{2}{|c|}{ Principal results } & \multirow{2}{*}{$\begin{array}{l}\text { Methodological } \\
\text { limitations } \\
\text { according to the } \\
\text { authors }\end{array}$} \\
\hline & & & & & $\begin{array}{l}\text { Positive } \\
\text { association } \\
\text { with frailty }\end{array}$ & $\begin{array}{c}\text { Inverse } \\
\text { association } \\
\text { with frailty }\end{array}$ & \\
\hline $\begin{array}{l}\text { Masel et al. } 26 \\
\text { (2009) }\end{array}$ & Cross-sectional & $\begin{array}{l}\text { Demographic: age, } \\
\text { gender, marital } \\
\text { status } \\
\text { Socioeconomic: } \\
\text { schooling, difficulty } \\
\text { paying expenses } \\
\text { Diseases and } \\
\text { health conditions: } \\
\text { CVD, stroke, } \\
\text { arthrosis, SAH, } \\
\text { diabetes mellitus, } \\
\text { fractures, number } \\
\text { of comorbidities } \\
\text { Nutritional status: } \\
\text { BMI } \\
\text { Other: quality } \\
\text { of life }\end{array}$ & Bivariate analysis & - & $\begin{array}{c}\text { Age, chronic } \\
\text { diseases, arthritis, } \\
\text { underweight } \\
\text { (BMI), low quality } \\
\text { of life }\end{array}$ & $\begin{array}{c}\text { Married, } \\
\text { schooling, } \\
\text { overweight (BMI) }\end{array}$ & $\begin{array}{l}\text { Cross-sectional } \\
\text { study, does not } \\
\text { allow causal } \\
\text { inferences; ethnic } \\
\text { homogeneity; } \\
\text { some variables } \\
\text { self-reported }\end{array}$ \\
\hline $\begin{array}{l}\text { Ottenbacher } \\
\text { et al. } 41 \text { (2009) }\end{array}$ & Longitudinal & $\begin{array}{c}\text { Demographic: age, } \\
\text { gender, marital } \\
\text { status } \\
\text { Socioeconomic: } \\
\text { financial status, } \\
\text { schooling } \\
\text { Diseases and } \\
\text { health conditions: } \\
\text { CVD, stroke, } \\
\text { arthritis, cancer, } \\
\text { diabetes mellitus } \\
\text { Lifestyle: smoking } \\
\text { Nutritional status: } \\
\text { weight, height } \\
\text { Psycho-behavioral: } \\
\text { cognitive function } \\
\text { and depressive } \\
\text { symptoms } \\
\text { Other: functional } \\
\text { status (ADL, IADL) }\end{array}$ & $\begin{array}{c}\text { Multiple linear } \\
\text { regression }\end{array}$ & All, in 3 models & $\begin{array}{l}\text { Age, history } \\
\text { of smoking, } \\
\text { diabetes mellitus, } \\
\text { arthritis, BMI, } \\
\text { depression, } \\
\text { number of } \\
\text { comorbidities }\end{array}$ & $\begin{array}{l}\text { Cognitive } \\
\text { function }\end{array}$ & $\begin{array}{l}\text { Conditions and } \\
\text { comorbidities self- } \\
\text { reported; original } \\
\text { sample excludes } \\
\text { individuals that } \\
\text { did not complete } \\
\text { the performance } \\
\text { tests required to } \\
\text { calculate frailty } \\
\text { construct. Persons } \\
\text { that remained } \\
\text { in the study } \\
\text { represented the } \\
\text { healthier members } \\
\text { of the original } \\
\text { sample }\end{array}$ \\
\hline $\begin{array}{l}\text { Szanton et } \\
\text { al. } 36 \text { (2009) }\end{array}$ & Cross-sectional & $\begin{array}{l}\text { Demographic: } \\
\text { age, race } \\
\text { Socioeconomic: } \\
\text { schooling } \\
\text { Diseases and } \\
\text { health conditions: } \\
\text { number of } \\
\text { chronic diseases } \\
\text { Nutritional status: } \\
\text { BMI } \\
\text { Lifestyle: smoking }\end{array}$ & Bivariate analysis & - & Age, BMI & Schooling & $\begin{array}{l}\text { Cross-sectional } \\
\text { study, does not } \\
\text { allow causal } \\
\text { inferences }\end{array}$ \\
\hline
\end{tabular}

(continues) 
Table 2 (continued)

\begin{tabular}{|c|c|c|c|c|c|c|c|}
\hline \multirow[t]{2}{*}{ Article (year) } & \multirow[t]{2}{*}{ Study design } & \multirow{2}{*}{$\begin{array}{c}\text { Independent } \\
\text { variables }\end{array}$} & \multirow{2}{*}{$\begin{array}{l}\text { Statistical } \\
\text { technique }\end{array}$} & \multirow{2}{*}{$\begin{array}{l}\text { Variables used } \\
\text { in adjustment } \\
\text { of final model }\end{array}$} & \multicolumn{2}{|c|}{ Principal results } & \multirow{2}{*}{$\begin{array}{l}\text { Methodological } \\
\text { limitations } \\
\text { according to the } \\
\text { authors }\end{array}$} \\
\hline & & & & & $\begin{array}{l}\text { Positive } \\
\text { association } \\
\text { with frailty }\end{array}$ & $\begin{array}{l}\text { Inverse } \\
\text { association } \\
\text { with frailty }\end{array}$ & \\
\hline $\begin{array}{l}\text { Wu et al. } 42 \\
\text { (2009) }\end{array}$ & Cross-sectional & $\begin{array}{c}\text { Demographic: } \\
\text { age } \\
\text { Diseases and } \\
\text { health conditions: } \\
\text { SAH, diabetes } \\
\text { mellitus, CHF, } \\
\text { osteoarthritis } \\
\text { Psycho- } \\
\text { behavioral: } \\
\text { depression } \\
\text { Nutritional status: } \\
\text { BMI, waist/hips } \\
\text { ratio } \\
\text { Lifestyle: smoking }\end{array}$ & $\begin{array}{l}\text { Bivariate analysis } \\
\text { and logistic } \\
\text { regression }\end{array}$ & - & $\begin{array}{c}\text { Age, depression, } \\
\text { waist } / \mathrm{hips} \text { ratio (> } \\
95 \mathrm{~cm})\end{array}$ & - & $\begin{array}{l}\text { Cross-sectional } \\
\text { study, does not } \\
\text { allow causal } \\
\text { inferences; sample } \\
\text { considered small }\end{array}$ \\
\hline $\begin{array}{l}\text { Alcalá et al. } 18 \\
\text { (2010) }\end{array}$ & Cross-sectional & $\begin{array}{l}\text { Demographic: } \\
\text { age, gender, } \\
\text { marital status } \\
\text { Socioeconomic: } \\
\text { schooling } \\
\text { Diseases and } \\
\text { health conditions: } \\
\text { chronic diseases } \\
\text { Other: functional } \\
\text { status (ADL, } \\
\text { IADL) }\end{array}$ & $\begin{array}{l}\text { Logistic } \\
\text { regression }\end{array}$ & $\begin{array}{l}\text { Age, schooling, } \\
\text { comorbidities, } \\
\text { functional } \\
\text { incapacity }\end{array}$ & $\begin{array}{l}\text { Age (> } 85 \text { years), } \\
\text { comorbidities, } \\
\text { functional } \\
\text { incapacity }\end{array}$ & Schooling & $\begin{array}{c}\text { Advanced age of } \\
\text { participants (> } 71 \\
\text { years), different } \\
\text { socioeconomic } \\
\text { characteristics } \\
\text { and lack of } \\
\text { homogeneity in } \\
\text { measurement of } \\
\text { frailty criteria may } \\
\text { have influenced } \\
\text { the observed } \\
\text { differences }\end{array}$ \\
\hline $\begin{array}{l}\text { Chang et al. } 37 \\
(2010)\end{array}$ & Cross-sectional & $\begin{array}{l}\text { Demographic: } \\
\text { age, race } \\
\text { Socioeconomic: } \\
\text { schooling } \\
\text { Diseases and } \\
\text { health conditions: } \\
\text { CKD, lung } \\
\text { disease, CVD, } \\
\text { diabetes mellitus, } \\
\text { anemia, arthritis, } \\
\text { peripheral } \\
\text { artery disease, } \\
\text { total number of } \\
\text { inflammatory } \\
\text { diseases } \\
\text { Psycho- } \\
\text { behavioral: } \\
\text { depressive } \\
\text { symptoms }\end{array}$ & $\begin{array}{l}\text { Multivariate } \\
\text { logistic } \\
\text { regression }\end{array}$ & $\begin{array}{l}\text { Age, race, } \\
\text { schooling }\end{array}$ & $\begin{array}{l}\text { All diseases, } \\
\text { depressive } \\
\text { symptoms }\end{array}$ & - & $\begin{array}{l}\text { Cross-sectional } \\
\text { study, does not } \\
\text { allow causal } \\
\text { inferences; study } \\
\text { limited to sample } \\
\text { of women, cannot } \\
\text { make inferences } \\
\text { for men; sample } \\
\text { with higher } \\
\text { percentage of } \\
\text { black women } \\
\text { than the general } \\
\text { population; } \\
\text { imprecise estimate } \\
\text { of CKD due to the } \\
\text { diagnostic method } \\
\text { used }\end{array}$ \\
\hline
\end{tabular}

(continues) 
Table 2 (continued)

\begin{tabular}{|c|c|c|c|c|c|c|c|}
\hline \multirow[t]{2}{*}{ Article (year) } & \multirow[t]{2}{*}{ Study design } & \multirow{2}{*}{$\begin{array}{l}\text { Independent } \\
\text { variables }\end{array}$} & \multirow{2}{*}{$\begin{array}{l}\text { Statistical } \\
\text { technique }\end{array}$} & \multirow{2}{*}{$\begin{array}{l}\text { Variables used } \\
\text { in adjustment } \\
\text { of final model }\end{array}$} & \multicolumn{2}{|c|}{ Principal results } & \multirow{2}{*}{$\begin{array}{l}\text { Methodological } \\
\text { limitations } \\
\text { according to the } \\
\text { authors }\end{array}$} \\
\hline & & & & & $\begin{array}{l}\text { Positive } \\
\text { association } \\
\text { with frailty }\end{array}$ & $\begin{array}{l}\text { Inverse } \\
\text { association } \\
\text { with frailty }\end{array}$ & \\
\hline $\begin{array}{l}\text { Chen et al. } 28 \\
(2010)\end{array}$ & Cross-sectional & $\begin{array}{l}\text { Demographic: } \\
\text { age, gender, } \\
\text { marital status } \\
\text { Socioeconomic: } \\
\text { schooling } \\
\text { Diseases } \\
\text { and health } \\
\text { conditions: CVD, } \\
\text { gout, diabetes } \\
\text { mellitus, kidney } \\
\text { disease, COPD, } \\
\text { osteoarthritis, } \\
\text { osteoporosis, } \\
\text { arthritis, } \\
\text { osteoporosis, } \\
\text { ulcer, cancer, } \\
\text { SAH, cataract, } \\
\text { liver and gall } \\
\text { bladder disease, } \\
\text { number of } \\
\text { comorbidities, } \\
\text { geriatric } \\
\text { syndromes } \\
\text { Psycho- } \\
\text { behavioral: } \\
\text { depressive } \\
\text { symptoms } \\
\text { Other: functional } \\
\text { incapacity }\end{array}$ & Bivariate analysis & - & $\begin{array}{l}\text { Age, female } \\
\text { gender, absence } \\
\text { of spouse, } \\
\text { functional } \\
\text { incapacity, } \\
\text { comorbidity for } \\
\text { chronic diseases, } \\
\text { depression, } \\
\text { geriatric } \\
\text { syndromes }\end{array}$ & Schooling & - \\
\hline $\begin{array}{l}\text { Hubbard et } \\
\text { al. } 43 \text { (2010) }\end{array}$ & Cross-sectional & $\begin{array}{c}\text { Nutritional status: } \\
\text { BMI, WC }\end{array}$ & $\begin{array}{l}\text { Logistic } \\
\text { regression }\end{array}$ & $\begin{array}{l}\text { Age, gender, } \\
\text { schooling, } \\
\text { wealth/assets, } \\
\text { smoking }\end{array}$ & $\begin{array}{c}W C \geq 88 \mathrm{~cm} \text { in } \\
\text { women and } \\
\geq 102 \mathrm{~cm} \text { in men }\end{array}$ & - & $\begin{array}{l}\text { Adaptation of } \\
\text { scale from Fried } \\
\text { et al. } 2 \text { on criterion } \\
\text { for weight loss }\end{array}$ \\
\hline $\begin{array}{l}\text { Syddaal et } \\
\text { al. } 44 \text { (2010) }\end{array}$ & Longitudinal & $\begin{array}{l}\text { Demographic: } \\
\text { age, gender } \\
\text { Socioeconomic: } \\
\text { socioeconomic } \\
\text { status, schooling, } \\
\text { number of cars, } \\
\text { own home } \\
\text { Lifestyle: physical } \\
\text { activity, smoking, } \\
\text { alcohol use }\end{array}$ & $\begin{array}{l}\text { Multivariate } \\
\text { logistic } \\
\text { regression }\end{array}$ & $\begin{array}{l}\text { Age, comorbidity, } \\
\text { lifestyle, } \\
\text { socioeconomic } \\
\text { status, car }\end{array}$ & $\begin{array}{l}\text { In men: number } \\
\text { of cars, age }\end{array}$ & $\begin{array}{c}\text { In men: } \\
\text { schooling, own } \\
\text { home } \\
\text { In women: } \\
\text { without own } \\
\text { home }\end{array}$ & $\begin{array}{l}\text { Cross-sectional } \\
\text { study, does not } \\
\text { allow causal } \\
\text { inferences; did } \\
\text { not assess other } \\
\text { socioeconomic } \\
\text { variables like } \\
\text { income; analysis of } \\
\text { information bias: } \\
\text { age and behaviors } \\
\text { such as smoking } \\
\text { and social factors } \\
\text { such home } \\
\text { ownership suffer } \\
\text { neighborhood } \\
\text { influence }\end{array}$ \\
\hline
\end{tabular}

(continues) 
Table 2 (continued)

\begin{tabular}{|c|c|c|c|c|c|c|c|}
\hline \multirow[t]{2}{*}{ Article (year) } & \multirow[t]{2}{*}{ Study design } & \multirow{2}{*}{$\begin{array}{l}\text { Independent } \\
\text { variables }\end{array}$} & \multirow{2}{*}{$\begin{array}{l}\text { Statistical } \\
\text { technique }\end{array}$} & \multirow{2}{*}{$\begin{array}{l}\text { Variables used } \\
\text { in adjustment } \\
\text { of final model }\end{array}$} & \multicolumn{2}{|c|}{ Principal results } & \multirow{2}{*}{$\begin{array}{l}\text { Methodological } \\
\text { limitations } \\
\text { according to the } \\
\text { authors }\end{array}$} \\
\hline & & & & & $\begin{array}{l}\text { Positive } \\
\text { association } \\
\text { with frailty }\end{array}$ & $\begin{array}{c}\text { Inverse } \\
\text { association } \\
\text { with frailty }\end{array}$ & \\
\hline $\begin{array}{l}\text { Szanton et } \\
\text { al. } 38(2010)\end{array}$ & Cross-sectional & $\begin{array}{l}\text { Demographic: } \\
\text { age, race } \\
\text { Socioeconomics: } \\
\text { schooling, } \\
\text { income, number } \\
\text { of home, } \\
\text { poverty } \\
\text { Diseases } \\
\text { and health } \\
\text { conditions: } \\
\text { number of } \\
\text { chronic diseases } \\
\text { Lifestyle: } \\
\text { smoking }\end{array}$ & $\begin{array}{l}\text { Multinomial } \\
\text { logistic } \\
\text { regression }\end{array}$ & $\begin{array}{l}\text { Race, schooling, } \\
\text { smoking, state } \\
\text { of insecurity, } \\
\text { number of } \\
\text { chronic diseases }\end{array}$ & Black race & $\begin{array}{l}\text { Schooling, } \\
\text { income }\end{array}$ & $\begin{array}{l}\text { Cross-sectional } \\
\text { study, does not } \\
\text { allow causal } \\
\text { inferences; only } \\
\text { considered white } \\
\text { and black races }\end{array}$ \\
\hline $\begin{array}{l}\text { Drey et al. } 45 \\
\text { (2011) }\end{array}$ & Cross-sectional & $\begin{array}{l}\text { Demographic: } \\
\text { age, gender } \\
\text { Socioeconomic: } \\
\text { schooling } \\
\text { Psycho- } \\
\text { behavioral: } \\
\text { cognitive } \\
\text { function, } \\
\text { depressive } \\
\text { symptoms } \\
\text { Other: quality } \\
\text { of life }\end{array}$ & Bivariate analysis & - & $\begin{array}{l}\text { Number of } \\
\text { diseases, } \\
\text { depressive } \\
\text { symptoms }\end{array}$ & $\begin{array}{c}\text { Cognitive } \\
\text { function, quality } \\
\text { of life }\end{array}$ & - \\
\hline $\begin{array}{l}\text { Giménez et } \\
\text { al. } 46 \text { (2011) }\end{array}$ & Cross-sectional & $\begin{array}{c}\text { Demographic: } \\
\text { age, gender } \\
\text { Socioeconomic: } \\
\text { marital status, } \\
\text { schooling, } \\
\text { income, living } \\
\text { alone } \\
\text { Nutritional } \\
\text { status: not } \\
\text { malnourished / } \\
\text { malnourished } \\
\text { (MNA) } \\
\text { Diseases } \\
\text { and health } \\
\text { conditions: } \\
\text { comorbidity } \\
\text { Psycho- } \\
\text { behavioral: } \\
\text { depressive } \\
\text { symptoms } \\
\text { Other: functional } \\
\text { status (ADL, } \\
\text { IADL) }\end{array}$ & Bivariate analysis & - & $\begin{array}{l}\text { Female gender, } \\
\text { comorbidity, } \\
\text { depressive } \\
\text { symptoms, } \\
\text { functional } \\
\text { dependency, } \\
\text { malnutrition }\end{array}$ & - & $\begin{array}{l}\text { Cross-sectional } \\
\text { study, does not } \\
\text { allow causal } \\
\text { inferences }\end{array}$ \\
\hline
\end{tabular}

(continues) 
Table 2 (continued)

\begin{tabular}{|c|c|c|c|c|c|c|c|}
\hline \multirow[t]{2}{*}{ Article (year) } & \multirow[t]{2}{*}{ Study design } & \multirow{2}{*}{$\begin{array}{l}\text { Independent } \\
\text { variables }\end{array}$} & \multirow{2}{*}{$\begin{array}{l}\text { Statistical } \\
\text { technique }\end{array}$} & \multirow{2}{*}{$\begin{array}{l}\text { Variables used } \\
\text { in adjustment } \\
\text { of final model }\end{array}$} & \multicolumn{2}{|c|}{ Principal results } & \multirow{2}{*}{$\begin{array}{l}\text { Methodological } \\
\text { limitations } \\
\text { according to the } \\
\text { authors }\end{array}$} \\
\hline & & & & & $\begin{array}{l}\text { Positive } \\
\text { association } \\
\text { with frailty }\end{array}$ & $\begin{array}{c}\text { Inverse } \\
\text { association } \\
\text { with frailty }\end{array}$ & \\
\hline $\begin{array}{l}\text { Garcia-Garcia } \\
\text { et al. } 47 \text { (2011) }\end{array}$ & Cross-sectional & $\begin{array}{l}\text { Demographic: } \\
\text { age, gender } \\
\text { Socioeconomic: } \\
\text { conjugal status, } \\
\text { mean years } \\
\text { of schooling, } \\
\text { educational level } \\
\text { Diseases and } \\
\text { health conditions: } \\
\text { comorbidities } \\
\text { (SAH, diabetes } \\
\text { mellitus, CVD, } \\
\text { COPD, peptic } \\
\text { ulcer, fractures, } \\
\text { osteoporosis, } \\
\text { arthritis, } \\
\text { dementia, } \\
\text { Parkinson, cancer, } \\
\text { osteoporosis, } \\
\text { osteoarthritis, } \\
\text { kidney, thyroid, } \\
\text { and liver } \\
\text { symer: functional } \\
\text { status (ADL, } \\
\text { IADL) } \\
\text { cholesterol, } \\
\text { stroke) } \\
\text { Psycho- } \\
\text { behavioral: } \\
\text { cognitive } \\
\text { function, } \\
\text { depressive } \\
\text { syms }\end{array}$ & Bivariate analysis & - & $\begin{array}{c}\text { Age, CVD, } \\
\text { Parkinson, stroke, } \\
\text { dementia, COPD, } \\
\text { hip fracture, } \\
\text { depressive } \\
\text { symptoms, } \\
\text { functional } \\
\text { dependency, } \\
\text { cognitive } \\
\text { impairment }\end{array}$ & - & - \\
\hline $\begin{array}{l}\text { Hoeck et al. } 48 \\
\text { (2011) }\end{array}$ & Cross-sectional & $\begin{array}{l}\text { Demographic: } \\
\text { age, gender } \\
\text { Socioeconomic: } \\
\text { socioeconomic } \\
\text { status, schooling, } \\
\text { income familiar, } \\
\text { housing situation } \\
\text { Diseases and } \\
\text { health conditions: } \\
\text { comorbidities } \\
\text { Other: year of } \\
\text { interview }\end{array}$ & $\begin{array}{c}\text { Bivariate analysis } \\
\text { and multivariate } \\
\text { logistic } \\
\text { regression }\end{array}$ & $\begin{array}{l}\text { Age, gender, } \\
\text { comorbidity }\end{array}$ & $\begin{array}{l}\text { Comorbidities, } \\
\text { Wallonia } \\
\text { (Belgium), rented } \\
\text { housing }\end{array}$ & $\begin{array}{c}\text { More recent } \\
\text { interview year, } \\
\text { schooling, family } \\
\text { income }\end{array}$ & - \\
\hline
\end{tabular}

(continues) 
Table 2 (continued)

\begin{tabular}{|c|c|c|c|c|c|c|c|}
\hline \multirow[t]{2}{*}{ Article (year) } & \multirow[t]{2}{*}{ Study design } & \multirow{2}{*}{$\begin{array}{l}\text { Independent } \\
\text { variables }\end{array}$} & \multirow{2}{*}{$\begin{array}{l}\text { Statistical } \\
\text { technique }\end{array}$} & \multirow{2}{*}{$\begin{array}{l}\text { Variables used } \\
\text { in adjustment } \\
\text { of final model }\end{array}$} & \multicolumn{2}{|c|}{ Principal results } & \multirow{2}{*}{$\begin{array}{l}\text { Methodological } \\
\text { limitations } \\
\text { according to } \\
\text { the authors }\end{array}$} \\
\hline & & & & & $\begin{array}{l}\text { Positive } \\
\text { association } \\
\text { with frailty }\end{array}$ & $\begin{array}{l}\text { Inverse } \\
\text { association } \\
\text { with frailty }\end{array}$ & \\
\hline $\begin{array}{l}\text { Danon- } \\
\text { Hersch et al. } \\
49 \text { (2012) }\end{array}$ & Cross-sectional & $\begin{array}{l}\text { Demographic: } \\
\text { year of birth, } \\
\text { gender } \\
\text { Diseases } \\
\text { and health } \\
\text { conditions: } \\
\text { number } \\
\text { of chronic } \\
\text { diseases (CVD, } \\
\text { stroke, diabetes } \\
\text { mellitus, SAH, } \\
\text { cancer, chronic } \\
\text { respiratory } \\
\text { disease, } \\
\text { arthritis), } \\
\text { self-reported } \\
\text { disease } \\
\text { Other: } \\
\text { functional } \\
\text { status (ADL, } \\
\text { IADL) }\end{array}$ & $\begin{array}{l}\text { Bivariate } \\
\text { analysis and } \\
\text { multivariate } \\
\text { logistic } \\
\text { regression }\end{array}$ & $\begin{array}{l}\text { Gender, } \\
\text { components of } \\
\text { frailty }\end{array}$ & $\begin{array}{l}\text { Number of } \\
\text { chronic diseases, } \\
\text { diseases self- } \\
\text { reported (except } \\
\text { hypercholestero- } \\
\text { lemia) }\end{array}$ & $\begin{array}{l}\text { Functional } \\
\text { capacity, year } \\
\text { of birth }\end{array}$ & $\begin{array}{l}\text { Since the analysis } \\
\text { was from a cohort, } \\
\text { losses of information } \\
\text { may have occurred; } \\
\text { differences in } \\
\text { operationalization of } \\
\text { the components in } \\
\text { criterion from Fried } \\
\text { et al. } 2\end{array}$ \\
\hline $\begin{array}{l}\text { Lakey et al. } 50 \\
\text { (2012) }\end{array}$ & Longitudinal & $\begin{array}{c}\text { Psycho-behavioral: } \\
\text { depressive } \\
\text { symptoms }\end{array}$ & $\begin{array}{l}\text { Multinomial } \\
\text { logistic } \\
\text { regression }\end{array}$ & $\begin{array}{l}\text { Age, income, } \\
\text { schooling, race, } \\
\text { living alone, } \\
\text { BMI, self-rated } \\
\text { health, ADL, } \\
\text { smoking, alcohol } \\
\text { consumption, } \\
\text { hormone } \\
\text { replacement } \\
\text { therapy, SAH, } \\
\text { diabetes mellitus, } \\
\text { CVD, COPD, hip } \\
\text { fracture, falls, } \\
\text { arthritis, cancer, } \\
\text { stroke, number of } \\
\text { comorbidities }\end{array}$ & $\begin{array}{l}\text { Depressive } \\
\text { symptoms }\end{array}$ & - & $\begin{array}{l}\text { Lack of } \\
\text { information on } \\
\text { indication for } \\
\text { antidepressants, } \\
\text { dosage, and } \\
\text { treatment } \\
\text { adherence }\end{array}$ \\
\hline
\end{tabular}

(continues) 
Table 2 (continued)

\begin{tabular}{|c|c|c|c|c|c|c|c|}
\hline \multirow[t]{2}{*}{ Article (year) } & \multirow[t]{2}{*}{ Study design } & \multirow{2}{*}{$\begin{array}{c}\text { Independent } \\
\text { variables }\end{array}$} & \multirow{2}{*}{$\begin{array}{l}\text { Statistical } \\
\text { technique }\end{array}$} & \multirow{2}{*}{$\begin{array}{l}\text { Variables used } \\
\text { in adjustment } \\
\text { of final model }\end{array}$} & \multicolumn{2}{|c|}{ Principal results } & \multirow{2}{*}{$\begin{array}{l}\text { Methodological } \\
\text { limitations } \\
\text { according to the } \\
\text { authors }\end{array}$} \\
\hline & & & & & $\begin{array}{l}\text { Positive } \\
\text { association } \\
\text { with frailty }\end{array}$ & $\begin{array}{c}\text { Inverse } \\
\text { association } \\
\text { with frailty }\end{array}$ & \\
\hline $\begin{array}{l}\text { Bastos- } \\
\text { Barbosa et al. } \\
51 \text { (2012) }\end{array}$ & Cross-sectional & $\begin{array}{c}\text { Demographic: age, } \\
\text { gender, race } \\
\text { Socioeconomic: } \\
\text { schooling, income, } \\
\text { conjugal status, } \\
\text { work } \\
\text { Diseases and } \\
\text { health conditions: } \\
\text { blood pressure, } \\
\text { number of } \\
\text { diseases, } \\
\text { comorbidities } \\
\text { (SAH, dyslipidemia, } \\
\text { osteoporosis, } \\
\text { osteoarthritis, } \\
\text { diabetes mellitus, } \\
\text { hypothyroidism) } \\
\text { Psycho-behavioral: } \\
\text { cognitive function } \\
\text { Nutritional } \\
\text { status: BMI, waist } \\
\text { circumference }\end{array}$ & $\begin{array}{l}\text { Fixed-effects } \\
\text { linear models } \\
\text { and ANOVA }\end{array}$ & Age, gender & $\begin{array}{l}\text { Systolic and } \\
\text { diastolic } \\
\text { pressure, waist } \\
\text { circumference }\end{array}$ & - & $\begin{array}{l}\text { Selection of frail } \\
\text { and non-frail } \\
\text { participants } \\
\text { according } \\
\text { to specific } \\
\text { criteria, which } \\
\text { does not allow } \\
\text { extrapolating to } \\
\text { other elderly; } \\
\text { small sample: } \\
\text { results cannot be } \\
\text { extrapolated to } \\
\text { populations with } \\
\text { very different } \\
\text { living conditions }\end{array}$ \\
\hline $\begin{array}{l}\text { Jürschik et } \\
\text { al. } 52 \text { (2012) }\end{array}$ & Cross-sectional & $\begin{array}{l}\text { Demographic: } \\
\text { age, gender, race } \\
\text { Socioeconomic: } \\
\text { schooling, income, } \\
\text { conjugal status, } \\
\text { work, living alone } \\
\text { Diseases and } \\
\text { health conditions: } \\
\text { number of } \\
\text { comorbidities } \\
\text { Psycho-behavioral: } \\
\text { cognitive function, } \\
\text { depressive } \\
\text { symptoms, quality } \\
\text { of life, social } \\
\text { relations } \\
\text { Lifestyle: } \\
\text { smoking, alcohol } \\
\text { consumption } \\
\text { Nutritional status: } \\
\text { nutritional risk } \\
\text { (MNA), BMI, waist } \\
\text { circumference } \\
\text { Other: functional } \\
\text { status (ADL, IADL), } \\
\text { self-rated health }\end{array}$ & $\begin{array}{c}\text { Bivariate analysis } \\
\text { and logistic } \\
\text { regression }\end{array}$ & - & $\begin{array}{l}\text { Age, female } \\
\text { gender, conjugal } \\
\text { status widowed } \\
\text { or single, } \\
\text { smoking, alcohol } \\
\text { consumption, } \\
\text { number of } \\
\text { comorbidities, } \\
\text { functional } \\
\text { incapacity, } \\
\text { depressive } \\
\text { symptoms, } \\
\text { cognitive } \\
\text { decline, risk of } \\
\text { malnutrition, } \\
\text { visual } \\
\text { impairment, poor } \\
\text { self-rated health }\end{array}$ & $\begin{array}{c}\text { Social interaction, } \\
\text { quality of life }\end{array}$ & $\begin{array}{l}\text { Modification } \\
\text { of criterion for } \\
\text { unintentional } \\
\text { weight loss; cross- } \\
\text { sectional study, } \\
\text { does not allow } \\
\text { causal inferences }\end{array}$ \\
\hline
\end{tabular}

(continues) 
Table 2 (continued)

\begin{tabular}{|c|c|c|c|c|c|c|c|}
\hline \multirow[t]{2}{*}{ Article (year) } & \multirow[t]{2}{*}{ Study design } & \multirow{2}{*}{$\begin{array}{l}\text { Independent } \\
\text { variables }\end{array}$} & \multirow{2}{*}{$\begin{array}{l}\text { Statistical } \\
\text { technique }\end{array}$} & \multirow{2}{*}{$\begin{array}{l}\text { Variables used } \\
\text { in adjustment } \\
\text { of final model }\end{array}$} & \multicolumn{2}{|c|}{ Principal results } & \multirow{2}{*}{$\begin{array}{l}\text { Methodological } \\
\text { limitations } \\
\text { according to the } \\
\text { authors }\end{array}$} \\
\hline & & & & & $\begin{array}{l}\text { Positive } \\
\text { association } \\
\text { with frailty }\end{array}$ & $\begin{array}{c}\text { Inverse } \\
\text { association } \\
\text { with frailty }\end{array}$ & \\
\hline $\begin{array}{l}\text { Chang et al. } 53 \\
(2012)\end{array}$ & Cross-sectional & $\begin{array}{c}\text { Demographic: age, } \\
\text { gender } \\
\text { Socioeconomic: } \\
\text { Living alone } \\
\text { Diseases and } \\
\text { health conditions: } \\
\text { blood pressure, } \\
\text { number of } \\
\text { comorbidities, } \\
\text { diseases (SAH, } \\
\text { diabetes mellitus, } \\
\text { CVD, arthritis, } \\
\text { peptic ulcer) } \\
\text { Psycho-behavioral: } \\
\text { cognitive function, } \\
\text { depressive } \\
\text { symptoms, health- } \\
\text { related quality of } \\
\text { life, social relations }\end{array}$ & $\begin{array}{l}\text { Bivariate analysis } \\
\text { and multivariate } \\
\text { regression } \\
\text { analysis }\end{array}$ & $\begin{array}{l}\text { Age, number of } \\
\text { comorbidities, } \\
\text { living alone, falls } \\
\text { in previous year, } \\
\text { arthritis, peptic } \\
\text { ulcer, depression }\end{array}$ & $\begin{array}{l}\text { Age, number of } \\
\text { comorbidities, } \\
\text { living alone, } \\
\text { arthritis, peptic } \\
\text { ulcer, depression }\end{array}$ & $\begin{array}{l}\text { Components of } \\
\text { health-related } \\
\text { quality of life } \\
\text { scale, like } \\
\text { physical and } \\
\text { mental }\end{array}$ & $\begin{array}{l}\text { Cross-sectional } \\
\text { study, does not } \\
\text { allow causal } \\
\text { inferences; study } \\
\text { population } \\
\text { predominantly } \\
\text { urban, which } \\
\text { does not allow } \\
\text { extrapolating the } \\
\text { findings to the } \\
\text { rural population; } \\
\text { small sample of } \\
\text { frail elderly; lack of } \\
\text { specific cutoff points } \\
\text { for components } \\
\text { of the diagnostic } \\
\text { criterion for frailty } \\
\text { in the study } \\
\text { population; low } \\
\text { response rate due } \\
\text { to exclusion of many } \\
\text { comorbidities }\end{array}$ \\
\hline $\begin{array}{l}\text { Sousa et al. } 54 \\
(2012)\end{array}$ & Cross-sectional & $\begin{array}{l}\text { Demographic: age, } \\
\text { gender, race } \\
\text { Socioeconomic: } \\
\text { conjugal } \\
\text { status, family } \\
\text { arrangement, } \\
\text { household } \\
\text { situation, } \\
\text { schooling, } \\
\text { monthly income, } \\
\text { occupation } \\
\text { Diseases and } \\
\text { health conditions: } \\
\text { comorbidities, } \\
\text { chronic diseases } \\
\text { (SAH, diabetes } \\
\text { mellitus, CVD, } \\
\text { malignant tumors, } \\
\text { arthritis or } \\
\text { rheumatism, lung } \\
\text { diseases, stroke, } \\
\text { osteoporosis) } \\
\text { Psycho-behavioral: } \\
\text { cognitive function, } \\
\text { depressive } \\
\text { symptoms } \\
\text { Other: falls, } \\
\text { functional status } \\
\text { (ADL, IADL), self- } \\
\text { rated health }\end{array}$ & $\begin{array}{l}\text { Bivariate } \\
\text { analysis and } \\
\text { binary logistic } \\
\text { regression }\end{array}$ & $\begin{array}{c}\text { Socio- } \\
\text { demographic } \\
\text { factors, functional } \\
\text { status, chronic } \\
\text { diseases, self- } \\
\text { rated health }\end{array}$ & $\begin{array}{l}\text { Advanced age } \\
\text { osteoporosis, } \\
\text { stroke, } \\
\text { depression, falls, } \\
\text { presence of } \\
\text { comorbidities, } \\
\text { functional } \\
\text { dependency, } \\
\text { poor self-rated } \\
\text { health }\end{array}$ & - & $\begin{array}{l}\text { Cross-sectional } \\
\text { study, does not } \\
\text { allow causal } \\
\text { inferences }\end{array}$ \\
\hline
\end{tabular}

(continues) 
Table 2 (continued)

\begin{tabular}{|c|c|c|c|c|c|c|c|}
\hline \multirow[t]{2}{*}{ Article (year) } & \multirow[t]{2}{*}{ Study design } & \multirow{2}{*}{$\begin{array}{l}\text { Independent } \\
\text { variables }\end{array}$} & \multirow{2}{*}{$\begin{array}{l}\text { Statistical } \\
\text { technique }\end{array}$} & \multirow{2}{*}{$\begin{array}{l}\text { Variables used } \\
\text { in adjustment } \\
\text { of final model }\end{array}$} & & \multirow{2}{*}{$\begin{array}{l}\text { Methodological } \\
\text { limitations } \\
\text { according to the } \\
\text { authors }\end{array}$} \\
\hline & & & & & $\begin{array}{l}\text { Positive } \\
\text { association } \\
\text { with frailty }\end{array}$ & $\begin{array}{l}\text { Inverse } \\
\text { association } \\
\text { with frailty }\end{array}$ & \\
\hline $\begin{array}{l}\text { Neri et al. } 55 \\
\text { (2012) }\end{array}$ & Cross-sectional & $\begin{array}{l}\text { Demographic: } \\
\text { age, gender } \\
\text { Socioeconomic: } \\
\text { family income, } \\
\text { family } \\
\text { arrangement } \\
\text { Diseases and } \\
\text { health conditions: } \\
\text { number of } \\
\text { chronic diseases } \\
\text { (SAH, diabetes } \\
\text { mellitus, CVD, } \\
\text { cancer, arthritis } \\
\text { or rheumatism, } \\
\text { ischemia, stroke, } \\
\text { depression, } \\
\text { osteoporosis) } \\
\text { Psycho- } \\
\text { behavioral: } \\
\text { cognitive } \\
\text { function, } \\
\text { depressive } \\
\text { symptoms } \\
\text { Other: } \\
\text { perception of } \\
\text { social support, } \\
\text { social isolation }\end{array}$ & $\begin{array}{l}\text { Bivariate analysis } \\
\text { and univariate } \\
\text { and multivariate } \\
\text { logistic } \\
\text { regression }\end{array}$ & - & \multicolumn{2}{|c|}{$\begin{array}{l}\text { No factors significantly associated } \\
\text { with frailty were found }\end{array}$} & $\begin{array}{l}\text { Cross-sectional } \\
\text { study, does not } \\
\text { allow causal } \\
\text { inferences; } \\
\text { difficulty in } \\
\text { controlling each } \\
\text { variable's effect; } \\
\text { limitations in } \\
\text { design and sample } \\
\text { size; unequal } \\
\text { number of men } \\
\text { and women in } \\
\text { sample; lack of } \\
\text { information on } \\
\text { care }\end{array}$ \\
\hline $\begin{array}{l}\text { Schnittger et } \\
\text { al. } 56 \text { (2012) }\end{array}$ & Cross-sectional & $\begin{array}{l}\text { Demographic: } \\
\text { age, gender } \\
\text { Socioeconomic: } \\
\text { educational level, } \\
\text { living alone } \\
\text { Diseases and } \\
\text { health conditions: } \\
\text { age-adjusted } \\
\text { comorbidity } \\
\text { index } \\
\text { Psycho- } \\
\text { behavioral: } \\
\text { cognitive } \\
\text { function, } \\
\text { psychological } \\
\text { status } \\
\text { Nutritional status: } \\
\text { nutritional risk } \\
\text { (MNA), BMI } \\
\text { Other: functional } \\
\text { status (ADL, } \\
\text { IADL) }\end{array}$ & $\begin{array}{l}\text { Bivariate analysis } \\
\text { and Kaiser- } \\
\text { Meyer-Olkin test }\end{array}$ & - & $\begin{array}{c}\text { Psychological } \\
\text { distress } \\
\text { (mood, stress, } \\
\text { neuroticism, } \\
\text { and emotional } \\
\text { loneliness) }\end{array}$ & - & $\begin{array}{l}\text { Cross-sectional } \\
\text { study, does not } \\
\text { allow causal } \\
\text { inferences; } \\
\text { dimensions of } \\
\text { psychological } \\
\text { distress measured } \\
\text { are specific to the } \\
\text { study population, } \\
\text { and cannot be } \\
\text { generalized to } \\
\text { other population }\end{array}$ \\
\hline
\end{tabular}

(continues) 
Table 2 (continued)

\begin{tabular}{|c|c|c|c|c|c|c|c|}
\hline \multirow[t]{2}{*}{ Article (year) } & \multirow[t]{2}{*}{ Study design } & \multirow{2}{*}{$\begin{array}{l}\text { Independent } \\
\text { variables }\end{array}$} & \multirow{2}{*}{$\begin{array}{l}\text { Statistical } \\
\text { technique }\end{array}$} & \multirow{2}{*}{$\begin{array}{l}\text { Variables used } \\
\text { in adjustment } \\
\text { of final model }\end{array}$} & \multicolumn{2}{|c|}{ Principal results } & \multirow{2}{*}{$\begin{array}{l}\text { Methodological } \\
\text { limitations } \\
\text { according to the } \\
\text { authors }\end{array}$} \\
\hline & & & & & $\begin{array}{l}\text { Positive } \\
\text { association } \\
\text { with frailty }\end{array}$ & $\begin{array}{c}\text { Inverse } \\
\text { association } \\
\text { with frailty }\end{array}$ & \\
\hline $\begin{array}{l}\text { Casale- } \\
\text { Martínez et } \\
\text { al. } 57 \text { (2012) }\end{array}$ & Cross-sectional & $\begin{array}{l}\text { Socioeconomic: } \\
\text { educational } \\
\text { level, parents' } \\
\text { educational level, } \\
\text { conjugal status, } \\
\text { household assets } \\
\text { and situation, } \\
\text { socioeconomic } \\
\text { status, } \\
\text { employment } \\
\text { benefits, } \\
\text { occupational } \\
\text { history, friends } \\
\text { and family } \\
\text { members living } \\
\text { in the same } \\
\text { neighborhood, } \\
\text { financial support } \\
\text { Diseases and } \\
\text { health conditions: } \\
\text { childhood history } \\
\text { Psycho- } \\
\text { behavioral: } \\
\text { ability to make } \\
\text { important } \\
\text { decisions } \\
\text { Other: religion, } \\
\text { volunteer work, } \\
\text { abuse }\end{array}$ & $\begin{array}{l}\text { Multinomial } \\
\text { logistic } \\
\text { regression }\end{array}$ & - & $\begin{array}{l}\text { Not having } \\
\text { companion, not } \\
\text { making important } \\
\text { decisions, poor } \\
\text { economic status, } \\
\text { abuse }\end{array}$ & $\begin{array}{l}\text { Employment } \\
\text { benefits }\end{array}$ & - \\
\hline $\begin{array}{l}\text { Macuco et al. } \\
58(2012)\end{array}$ & Cross-sectional & $\begin{array}{l}\text { Demographic: } \\
\text { age, gender } \\
\text { Socioeconomic: } \\
\text { years of } \\
\text { schooling, } \\
\text { monthly family } \\
\text { income } \\
\text { Psycho- } \\
\text { behavioral: } \\
\text { cognitive } \\
\text { function, } \\
\text { loneliness, } \\
\text { adverse life } \\
\text { events } \\
\text { Other: functional } \\
\text { status (IADL) }\end{array}$ & $\begin{array}{l}\text { Bivariate analysis } \\
\text { and univariate } \\
\text { and multivariate } \\
\text { linear regression }\end{array}$ & - & Age & $\begin{array}{c}\text { Cognitive } \\
\text { function, years } \\
\text { of schooling, } \\
\text { monthly family } \\
\text { income }\end{array}$ & $\begin{array}{l}\text { Rigid exclusion } \\
\text { criteria; high } \\
\text { number of } \\
\text { younger elderly in } \\
\text { the ample; cross- } \\
\text { sectional study, } \\
\text { no follow-up of } \\
\text { cohort }\end{array}$ \\
\hline
\end{tabular}

ADL: activities of daily living; BMI: body mass index ; CHF: congestive heart failure; CKD: chronic kidney disease; COPD: chronic obstructive pulmonary disease; CVD: cardiovascular disease; IADL: instrumental activities of daily living; MNA: mini nutritional assessment; SAH: systemic arterial hypertension; WC: waist circumference.

Note: the variables and results presented here are those related to this study's objective. 
Table 3

Assessment of risk of bias according to adaptation of Newcastle-Ottawa Scale 13.

\begin{tabular}{|c|c|c|c|c|c|}
\hline Article (year) & $\begin{array}{c}\text { Independent } \\
\text { variables obtained }\end{array}$ & $\begin{array}{c}\text { Is the assessment } \\
\text { of frailty } \\
\text { adequate? }\end{array}$ & $\begin{array}{l}\text { Representativeness } \\
\text { of sample }\end{array}$ & $\begin{array}{l}\text { Participant } \\
\text { selection }\end{array}$ & $\begin{array}{c}\text { Definition of } \\
\text { control group or } \\
\text { cohort * }\end{array}$ \\
\hline Fried et al. 2 (2001) & B & B & । & B & । \\
\hline Newman et al. 27 (2001) & B & B & 1 & B & 1 \\
\hline Blaum et al. 33 (2005) & B & A & 1 & B & - \\
\hline Woods et al. 29 (2005) & B & A & 1 & B & I \\
\hline Hirsch et al. 25 (2006) & B & B & 1 & B & - \\
\hline Michelon et al. 17 (2006) & B & 1 & I & B & - \\
\hline Semba et al. 34 (2006) & B & 1 & 1 & B & I \\
\hline Ávila-Funes et al. 16 (2008) & B & A & । & B & 1 \\
\hline Alvarado et al. 39 (2008) & B & A & 1 & B & - \\
\hline Chaves et al. 35 (2008) & B & A & A & B & - \\
\hline Endeshaw et al. 40 (2009) & B & B & 1 & B & - \\
\hline Masel et al. 26 (2009) & B & 1 & 1 & B & - \\
\hline Ottenbacher et al. 41 (2009) & B & 1 & 1 & B & I \\
\hline Szanton et al. 36 (2009) & B & A & 1 & B & - \\
\hline Wu et al. 42 (2009) & B & A & 1 & B & - \\
\hline Alcalá et al. 18 (2010) & B & A & B & B & - \\
\hline Chang et al. 37 (2010) & B & A & I & B & - \\
\hline Chen et al. 28 (2010) & 1 & A & 1 & B & - \\
\hline Hubbard et al. 43 (2010) & I & A & B & B & - \\
\hline Syddaal et al. 44 (2010) & B & A & 1 & B & I \\
\hline Szanton et al. 38 (2010) & B & A & A & B & - \\
\hline Drey et al. 45 (2011) & B & B & 1 & B & - \\
\hline Giménez et al. 46 (2011) & B & A & 1 & B & - \\
\hline Garcia-Garcia et al. 47 (2011) & B & 1 & B & A & - \\
\hline Hoeck et al. 48 (2011) & I & A & B & B & - \\
\hline Danon-Hersch et al. 49 (2012) & B & A & B & B & - \\
\hline Lakey et al. 50 (2012) & B & A & 1 & B & B \\
\hline Bastos-Barbosa et al. 51 (2012) & B & B & 1 & B & - \\
\hline Jürschik et al. 52 (2012) & B & A & 1 & B & - \\
\hline Chang et al. 53 (2012) & B & A & 1 & B & - \\
\hline Sousa et al. 54 (2012) & B & B & 1 & B & - \\
\hline Neri et al. 55 (2012) & B & B & 1 & B & - \\
\hline Schnittger et al. 56 (2012) & B & 1 & 1 & B & - \\
\hline Casale-Martínez et al. 57 (2012) & I & 1 & 1 & B & - \\
\hline Macuco et al. 58 (2012) & B & B & 1 & B & - \\
\hline
\end{tabular}

Classification of items: B - low risk of bias; I - uncertain risk of bias; A - high risk of bias.

* Only for longitudinal studies.

\section{Discussion}

The principal socio-demographic, psycho-behavioral, health-related, nutritional, and lifestyle factors positively associated with frailty were: age, female gender, black race/color, cardiovascular diseases, number of comorbidities/ diseases, functional incapacity, poor self-rated health, depressive symptoms, BMI, and smok- ing. Inversely associated factors were schooling, income, cognitive function, and alcohol use.

Although the selected studies had different designs, sample sizes, and locations, they showed homogeneity in the relations between the demographic and socioeconomic variables and frailty. A longitudinal study of 5,317 North Americans over 65 years of age showed that prevalence of frailty was higher in the oldest old, women, 
Figure 2

Graph on risk of bias in selected studies.

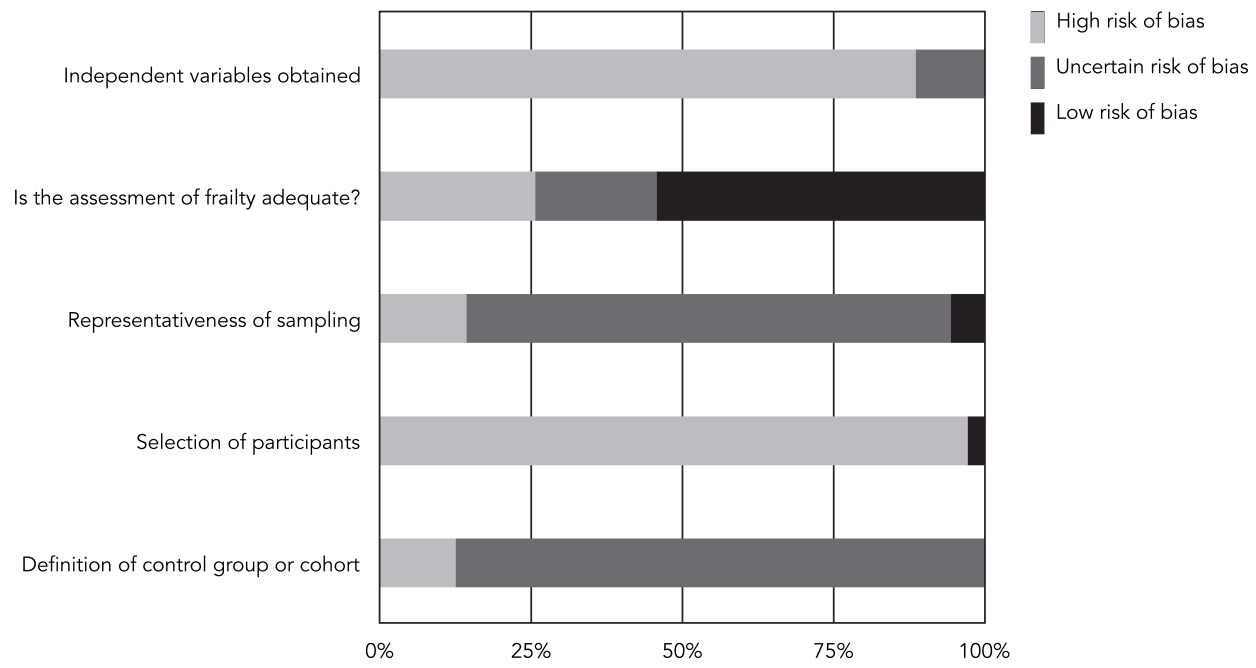

blacks, and low-income individuals 2 . This association was also found in a longitudinal study of French elderly 16 and in cross-sectional studies of both American and Spanish elderly 17,18. At more advanced ages there is an increase in prefrail and frail elderly, suggesting that frailty is a progressive condition; the phenomenon occurs more significantly after 80 years of age. One hypothesis for this relationship between increasing age and frailty lies in the cellular oxidative stress that accumulates over the years, modulated by exogenous and endogenous agents that influence the production of reactive oxygen species, leading to DNA damage. Such damage induces alterations at the cellular and systemic levels, with deregulations in the processes of inflammation, apoptosis, necrosis, and proliferation that result in various adverse conditions that increase over the years, such as loss of muscle mass (sarcopenia), diabetes, cancer, and frailty 19,20,21.

The higher prevalence of frailty in women can be explained by the greater physiological muscle mass loss in females during aging, in addition to their being more prone to the development of sarcopenia, an intrinsic risk for developing the frailty syndrome 5 . Other hypotheses included women's greater longevity and the fact that they show a higher prevalence of chronic illnesses than men 22 .

Race is a strong conditioning factor for health status, since blacks are at a disadvantage in relation to whites. Studies have shown that black race/color is an important indicator of low socioeconomic status and is associated with deficient health and high mortality risk 23,24 , contributing indirectly and directly to development of the syndrome. Furthermore, some authors believe that race is a marker for genetic polymorphisms that have an influence on the emergence of frailty 25 .

Income and schooling do not act directly in the pathophysiology of frailty, but interfere in the individual's lifestyle and quality of life and thus in factors that vary with socioeconomic status, including gender and age, which can influence the frailty process 25 .

As for diseases associated with frailty, CVD and the presence of two or more comorbidities are relevant for the occurrence of this syndrome in the elderly. In a cross-sectional study of 1,008 elderly Mexicans, self-reported chronic diseases such as CVD, hypertension, diabetes mellitus, and arthritis were associated with frailty 26 . A cross- sectional and longitudinal study in North Americans, but with diagnosis by clinical examination, showed an equivalent association 2,27 . Some researchers contend that CVD and some comorbidities are related to atherosclerosis, a chronic inflammatory state that can result in systemic catabolism and other pathophysiological changes, which can contribute to the clinical manifestations of frailty 2,27 . 
A direct association was also observed between functional incapacity and frailty. Recent cross-sectional studies in both Chinese and Spanish subjects showed that a major portion of the frail elderly show functional incapacity 18,28 . A longitudinal study in a robust sample of 5,317 elderly also showed this relationship 2 . The authors contend that frailty can be a precursor of functional incapacity. However, one cannot overlook the possibility of reverse causality between functional capacity and frailty.

In the area of psycho-behavioral variables, decreased cognitive function and the presence of depressive symptoms have been related to frailty. Studies with different samples, (American, Mexican, and French elderly) showed increased prevalence of frailty in elders submitted to different questionnaires with scales for depression or cognitive function and that presented depressive symptoms or cognitive impairment according to the tests 2,16,18. Elderly with cognitive impairment probably experience greater difficulty in eating, exercising, and walking, which can lead to weight loss and decreased motor function and favor the syndrome's onset and progression. As for depressive symptoms, the literature shows that the relationship to frailty is biologically plausible, since depressed persons normally present weight loss, limited activity, and isolation, thus predisposing to progressive loss of muscle mass and strength, conditions that accelerate the establishment of the syndrome 5 .

As for nutritional and lifestyle variables, underweight elderly according to BMI and those with a higher proportion of overweight according to BMI showed a higher prevalence of frailty. In a study of elderly Mexicans, Masel et al. 26 found that underweight was related to frailty. However, although Woods et al. 29 found the same association, they showed that frailty could also be associated with overweight and obesity. The association between frailty and underweight may be related to the common loss of muscle mass in individuals with unintentional weight loss 30 . Meanwhile, the relationship between frailty and overweight and obesity may be due to the fact that excess weight can be associated with activation of inflammatory processes, which trigger systemic alterations, which in turn can influence the onset of frailty 5 . Still other authors speculate that weight extremes in the elderly are related to loss of muscle mass in arms and legs, and that the phenomenon of "sarcopenic obesity", referring to weight gain concurrent with loss of muscle mass, leads to difficulty in mobility, reduced strength, and thus physical inactivity, one of the elements in the frailty cycle 31,32 .
As for studies that showed an inverse association between alcohol consumption and frailty $16,17,29$ and a positive association between smoking and the syndrome 17,29 , the authors do not discuss the possible explanations for such findings. Woods et al. 29 merely mention that when stratifying the variable in doses per week, elders with moderate alcohol consumption had 13 to $31 \%$ lower odds of presenting frailty syndrome, even after adjusting for chronic diseases that have been associated with moderate alcohol use. Caution has been suggested when analyzing such associations, especially those related to alcohol consumption, since not all the studies conducted regression analyses or adjusted for potential confounders when cross-analyzing such variables.

Since 9 of the 35 studies (26\%) only included women, a comparison was made been factors associated with frailty in both genders and in studies only with females, showing that there was no difference between the associated factors, suggesting that the elder's gender does not have a decisive weight in the establishment of the syndrome.

Importantly, the studies varied in both their design and the methods used to measure the independent variables. Most adopted a cross-sectional design, which does not allow establishing a cause-and-effect relationship between the independent variables and the outcome. In addition, 18 studies performed bivariate analyses, and a total of 16 did not adjust for potential confounders. However, in general such limitations appear not to have influenced the associations, considering the consistency between results.

As for assessment of risk of bias, a question that called considerable attention was the adequacy of the diagnostic assessment of frailty. As mentioned, the choice of the criterion adopted by Fried et al. 2 (among various existing criteria) to assess frailty in this review was due to the lack of a consensus or gold standard for identifying the syndrome and to the fact that Fried's definition is widely used in other Brazilian and international scientific studies. The current review did not aim to critically discuss the instruments for evaluating frailty proposed by the literature, so the analysis of bias in the assessment of frailty merely aimed to verify the extent to which the studies analyzed in the sample deviated from the original proposal by Fried et al. 2 .

Focusing on this point, we found that only $36 \%$ of the studies assessed frailty comprehensively as Fried et al. 2 proposed, and that $74 \%$ performed some modification of the five components. Changes in proposed criteria can lead to erroneous conclusions when comparing the 
results to those of other studies. Meanwhile, the instruments proposed to assess some component, as for example the questionnaire proposed by Fried et al. 2 to estimate low level of physical activity, may contain items that do not agree with the study's local reality, which would probably lead the authors to adapt the questionnaire to obtain a more adequate and true response. Furthermore, other authors adopted other criteria and validated such changes in relation to the proposal by Fried et al. 2, while still others did not conduct a validation process or failed to report it. Thus, common sense is recommended when analyzing articles for comparison with data from other authors.

In addition, some studies drawing on the same cohort 17,33,34,35,36,37,38 used different descriptions of the instruments used to measure the component of the criterion used by Fried et al. 2, which raises doubts in their analysis.

This review presents some limitations. First, by limiting the languages of the publications to English, Portuguese, or Spanish and the databases for the article search, some relevant study may have been left out. The second relates to the limiting the diagnostic criterion for frailty according to Fried et al. ${ }^{2}$. The scientific literature provides different instruments with various markers, which are being tested in international studies. Thus, some outstanding studies may have been lost. Another limitation relates to restricting the presentation of results to those with statistical significance. This decision was due to the fact that one cannot reach conclusions on associations that are not statistically significant, and due to the number and scope of the target variables.

\section{Final remarks}

The worldwide increase in prevalence of frailty among the elderly raises challenges for all countries. Knowledge of the factors associated with the syndrome and the complexity of its determinants helps formulate measures for prevention and early intervention, thus fostering aging with better quality of life and greater dignity. Although the studies and their comparison present limitations, this review highlights a series of socio-demographic, psycho-behavioral, health-related, and nutritional factors that assist the identification of more vulnerable groups and that are amenable to intervention.

Importantly, although demographic determinants showed a relationship to frailty in the elderly, some determinants are not subject to changes and interventions. For example, it is impossible to alter age or gender, but they should be considered anyway, since various health conditions increase with age and occur differently between men and women.

Thus, planning of individual and collective health measures for the elderly should consider the factors identified here as related to the frailty syndrome, such as: age, black race/color, female gender, CVD, number of comorbidities/diseases, functional incapacity, poor self-rated health, depressive symptoms, BMI, smoking, schooling, income, cognitive function, and alcohol consumption (the latter with caution). It is also important to investigate other factors not explored in this review, besides conducting meta-analyses aimed at a critical assessment of the evidence and a discussion of the possible heterogeneity of results, in addition to an analysis of the strength of available evidence on the association found between a given variable and frailty in order to better understand how the way of living can interfere in the way of aging and favor the establishment of this syndrome. 


\section{Resumen}

La fragilidad es un síndrome que causa daño en la vida práctica de ancianos, ya que está relacionada con un mayor riesgo de dependencia, caídas, hospitalización, institucionalización y muerte. El objetivo de esta revisión sistemática fue identificar factores sociodemográficos, psicoconductuales, de condiciones de salud, nutrición y estilo de vida asociados a fragilidad en ancianos. Se detectaron 4.183 estudios publicados entre 2001 y 2013 en las bases bibliográficas y se seleccionaron 182 artículos completos. Después de la lectura y aplicación de los criterios de selección, quedaron 35 artículos elegibles para análisis. Los principales factores asociados fueron edad, sexo femenino, raza negra, educación, ingresos, enfermedad cardiovascular, número de comorbilidades/enfermedad, incapacidad funcional, autopercepción de mala salud, síntomas depresivos, función cognitiva, índice de masa corporal, tabaquismo y consumo de alcohol. El conocimiento de la complejidad de los determinantes de fragilidad ayuda en la formulación de medidas preventivas e intervención temprana, asegurando mejor calidad de vida.

Anciano Frágil; Calidad de Vida; Factores de Riesgo

\section{Contributors}

A. C. Mello participated in all stages of the article's elaboration. E. M. Engstrom and L. C. Alves contributed to the literature review, article selection, and data extraction, besides participating in writing and critically revising the article.

\section{Acknowledgments}

The authors wish to thank librarian Gizele da Rocha Ribeiro for her outstanding contribution to defining the search strategies and Capes for granting the $\mathrm{PhD}$ scholarship to A. C. Mello.

\section{References}

1. Morley JE, Vellas B, van Kan GA, Anker SD, Bauer JM, Bernabei R, et al. Frailty consensus: a call to action. J Am Med Dir Assoc 2013; 14:392-7.

2. Fried LP, Tangen CM, Walston J, Newman AB, Hirsch C, Gottdiener J, et al. Frailty in older adults: evidence for a phenotype. J Gerontol A Biol Sci Med Sci 2001; 56:M146-56.

3. Fried LP, Ferrucci L, Darer J, Williamson JD, Anderson G. Untangling the concepts of disability, frailty, and comorbidity: implications for improved targeting and care. J Gerontol A Biol Sci Med Sci 2004; 59:M255-63

4. Ministério da Saúde. Envelhecimento e saúde da pessoa idosa. Brasília: Ministério da Saúde; 2006. (Cadernos de Atenção Básica, Normas e Manuais Técnicos, 19).

5. Espinoza SE, Fried LP. Risk factors for frailty in the older adult. Clin Geriatr 2007; 15:37.

6. Alves LC, Leimann BCQ, Vasconcelos MEL, Carvalho MS, Vasconcelos AGG, Fonseca TCO, et al. A influência das doenças crônicas na capacidade funcional dos idosos do Município de São Paulo, Brasil. Cad Saúde Pública 2007; 23:1924-30.

7. Rockwood K. Mitnitski A. Frailty in relation to the accumulationof deficits. J Gerontol Med Sci 2007 62:722-7.

8. Levers MJ, Estabrooks CA, Kerr JCR. Factors contributing to frailty: literature review. J Adv Nurs 2006; 56:282-91.

9. Kaiser MJ, Bandinelli S, Lunenfeld B. Frailty and the role of nutrition in older people: a review of the current literature. Acta Biomed 2010; 81 Suppl 1:37-45.

10. Orme J, Reis J, Herz E. Factorial and discriminate validity of the Center for Epidemiological Studies depression (CES-D) scale. J Clin Psychol 1986; 42:28-33.

11. Taylor HL, Jacobs Jr. DR, Schucker B, Knudsen J, Leon AS, Debacker G. A questionnaire for the assessment of leisure-time physical activities. J Chronic Dis 1978; 31:745-55.

12. Higgins JPT, Altman DG. Chapter 8: assessing risk of bias in included studies. In: Higgins JPT, Green S, editors. Cochrane Handbook for Systematic Reviews of Interventions, Version 5.0.1. The Cochrane Collaboration; 2008. http://www.cochrane-handbook.org (accessed on 20/May/2013). 
13. Wells GA, Shea B, O'Connell D, Peterson J, Welch V, Losos M, Tugwell P. The Newcastle-Ottawa Scale (NOS) for assessing the quality of nonrandomised studies in meta-analyses. http://www.ohri.ca/pro grams/clinical_epidemiology/oxford.asp (accesssed on 20/May/2013).

14. Stang A. Critical evaluation of the Newcastle-Ottawa scale for the assessment of the quality of nonrandomized studies in meta-analyses. Eur J Epidemiol 2010; 25:603-5.

15. Folstein MF, Folstein SE, McHugh PR. Mini-Mental State: a practical method for grading the cognitive state of patients for the clinician. J Psychiatr Res 1975; 12:189-98.

16. Ávila-Funes JA, Helmer C, Amieva H, BarbergerGateau P, Le Goff M, Ritchie K, et al. Frailty among community-dwelling elderly people in France: the three-city study. J Gerontol A Biol Sci Med Sci 2008; 63:1089-96.

17. Michelon E, Blaum C, Semba RD, Xue QL, Ricks MO, Fried LP. Vitamin and carotenoid status in older women: associations with the frailty syndrome. J Gerontol A Biol Sci Med Sci 2006; 61:600-7.

18. Alcalá MVC, Puime ÁO, Santos MTS, Barral AG, Montalvo JIG, Zunzunegui MV. Prevalencia de fragilidad en una población urbana de mayores de 65 años y su relación con comorbilidad y discapacidad. Aten Primaria 2010; 42:520-7.

19. Mulero J, Zafrilla P, Martinez-Cacha A. Oxidative stress, frailty and cognitive decline. J Nutr Health Aging 2011; 15:756-60.

20. Karahalil B, Bohr VA, Wilson diabetes mellitus. Impact of DNA polymorphisms in key DNA base excision repair proteins on cancer risk. Hum Exp Toxicol 2012; 31:981-1005.

21. Cutler RG. Human longevity and aging: possible role of reactive oxygen species. Ann N Y Acad Sci 1991; 621:1-28.

22. Pinheiro RS, Viacava F, Travassos C, Brito AS. Gênero, morbidade, acesso e utilização de serviços de saúde no Brasil. Ciênc Saúde Coletiva 2002; 7:687-707.

23. Fiorio NM, Flor LS, Padilha M, Castro DS, Molina MCB. Mortalidade por raça/cor: evidências de desigualdades sociais em Vitória (ES), Brasil. Rev Bras Epidemiol 2011; 14:522-30.

24. Batista LE. Masculinidade, raça/cor e saúde. Ciênc Saúde Coletiva 2005; 10:71-80.

25. Hirsch C, Anderson ML, Newman A, Kop W, Jackson S, Gottdiener J, et al. The association of race with frailty: The Cardiovascular Health Study. Ann Epidemiol 2006; 16:545-53.

26. Masel MC, Graham JE, Reistetter TA, Markides KS, Ottenbacher KJ. Frailty and health related quality of life in older Mexican Americans. Health Qual Life Outcomes 2009; 7:70.

27. Newman AB, Gottdiener JS, McBurnie MA, Hirsch $\mathrm{CH}$, Willem JK, Tracy R, et al. Associations of subclinical cardiovascular disease with frailty. J Gerontol A Biol Sci Med Sci 2001; 56:M158-66.

28. Chen CY, Wu SC, Chen LJ, Lue BH. The prevalence of subjective frailty and factors associated with frailty in Taiwan. Arch Gerontol Geriatr 2010; 50:S43-7.
29. Woods NF, LaCroix AZ, Gray SL, Aragaki A, Cochrane BB, Brunner RL, et al. Frailty: emergence and consequences in women aged 65 and older in the Women's Health Initiative Observational Study. J Am Geriatr Soc 2005; 53:1321-30.

30. Chemin SS, Mura JDP. Tratado de alimentação, nutrição e dietoterapia. São Paulo: Roca; 2007.

31. Roubenoff R. Sarcopenic obesity. Does muscle loss cause fat gain? Lessons from rheumatoid arthritis and osteoarthritis. Ann N Y Acad Sci 2000; 904:553-7.

32. Jenkins KR. Obesity's effects on the onset of functional impairment among older adults. Gerontologist 2004; 44:206-16.

33. Blaum CS, Xue QL, Michelon E, Semba RD, Fried LP. The association between obesity and the frailty syndrome in older women: The Women's Health and Aging Studies. J Am Geriatr Soc 2005; 53:92734 .

34. Semba RD, Bartali B, Zhou J, Blaum C, Ko CW, Fried LP. Low serum micronutrient concentrations predict frailty among older women living in the community. J Gerontol A Biol Sci Med Sci 2006; 61:594-9.

35. Chaves PH, Varadhan R, Lipsitz LA, Stein PK, Windham BG, Tian J, et al. Physiological complexity underlying heart rate dynamics and frailty status in community-dwelling older women. J Am Geriatr Soc 2008; 56:1698-703.

36. Szanton SL, Allen JK, Seplaki CL, Bandeen-Roche K, Fried LP. Allostatic load and frailty in the women's health and aging studies. Biol Res Nurs 2009; 10:248-56.

37. Chang SS, Weiss CO, Xue QL, Fried LP. Patterns of comorbid inflammatory diseases in frail older women: the Women's Health and Aging Studies I and II. J Gerontol A Biol Sci Med Sci 2010; 65: 407-13.

38. Szanton SL, Seplaki CL, Thorpe RJ, Allen JK, Fried LP. Socioeconomic status is associated with frailty: the Women's Health and Aging Studies. J Epidemiol Community Health 2010; 64:63-7.

39. Alvarado BE, Zunzunegui MV, Béland F, Bamvita JM. Life course social and health conditions linked to frailty in Latin American older men and women. J Gerontol A Biol Sci Med Sci 2008; 63:1399-406.

40. Endeshaw YW, Unruh ML, Kutner M, Newman AB, Bliwise DL. Sleep-disordered breathing and frailty in the Cardiovascular Health Study Cohort. Am J Epidemiol 2009; 170:193-202.

41. Ottenbacher KJ, Graham JE, Al Snih S, Raji M, Samper-Ternent R, Ostir GV, et al. Mexican Americans and frailty: findings from the hispanic established populations epidemiologic studies of the elderly. Am J Public Health 2009; 99:673-9.

42. Wu I, Shiesh SC, Kuo PH, Lin XZ. High oxidative stress is correlated with frailty in elderly chinese. J Am Geriatr Soc 2009; 57:1666-71.

43. Hubbard RE, Lang IA, Llewellyn DJ, Rockwood K. Frailty, body mass index, and abdominal obesity in older people. J Gerontol A Biol Sci Med Sci 2010; 65:377-81. 
44. Syddall H, Roberts HC, Evandrou M, Cooper C, Bergman H, Sayer AA. Prevalence and correlates of frailty among community-dwelling older men and women: findings from the Hertfordshire Cohort Study. Age Ageing 2010; 39:197-203.

45. Drey M, Wehr H, Wehr G, Uter W, Lang F, Rupprecht $\mathrm{R}$, et al. The frailty syndrome in general practitioner care. Z Gerontol Geriatr 2011; 44:48-54.

46. Giménez PJ, Bravo MAE, Orrio CN, Satorra TB. Criterios de fragilidad del adulto mayor. Estudio piloto. Aten Primaria 2011; 43:190-6.

47. Garcia-Garcia FJ, Avila GG, Alfaro-Acha A, Andres MA, Aparicio ME, Aparicio SH, et al. The prevalence of frailty syndrome in an older population from Spain. The Toledo Study for Healthy Aging. J Nutr Health Aging 2011; 15:852-6.

48. Hoeck S, François G, Geerts J, Van der Heyden J, Vandewoude M, Van Hal G. Health-care and home-care utilization among frail elderly persons in Belgium. Eur J Public Health 2012; 22:671-7.

49. Danon-Hersch N, Rodondi N, Spagnoli J, SantosEggimann B. Prefrailty and chronic morbidity in the youngest old: an insight from the Lausanne cohort Lc65+. J Am Geriatr Soc 2012; 60:1687-94.

50. Lakey SL, LaCroix AZ, Gray SL, Borson S, Williams CD, Calhoun D, et al. Antidepressant use, depressive symptoms, and incident frailty in women aged 65 and older from the Women's Health Initiative Observational Study. J Am Geriatr Soc 2012; 60:854-61.

51. Bastos-Barbosa RG, Ferriolli E, Coelho EB, Moriguti JC, Nobre F, Lima NKC. Association of frailty syndrome in the elderly with higher blood pressure and other cardiovascular risk factors. Am J Hypertens 2012; 25:1156-61.
52. Jürschik P, Nunin C, Botigué T, Escobar MA, Lavedán A, Viladrosa M. Prevalence of frailty and factors associated with frailty in the elderly population of Lleida, Spain: the FRALLE survey. Arch Gerontol Geriatr 2012; 55:625-31.

53. Chang YW, Chen WL, Lin FG, Fang WH, Yen MY, Hsieh CC, et al. Frailty and its impact on healthrelated quality of life: a cross-sectional study on elder community-dwelling preventive health service users. PLoS One 2012; 7:e38079.

54. Sousa ACPA, Dias RC, Maciel ACC, Guerra RO. Frailty syndrome and associated factors in community-dwelling elderly in Northeast Brazil. Arch Gerontol Geriatr 2012; 54:e95-101.

55. Neri AL, Yassuda MS, Fortes-Burgos ACG, Mantovani EP, Arbex FS, Torres SVS, et al. Relationships between gender, age, family conditions, physical and mental health, and social isolation of elderly caregivers. Int Psychogeriatr 2012; 24:472.

56. Schnittger RI, Walsh CD, Casey AM, Wherton JP, McHugh JE, Lawlor BA. Psychological distress as a key component of psychosocial functioning in community-dwelling older people. Aging Ment Health 2012; 16:199-207.

57. Casale-Martínez RI, Navarrete-Reyes AP, ÁvilaFunes JA. Social determinants of frailty in elderly Mexican community-dwelling adults. J Am Geriatr Soc 2012; 60:800-2.

58. Macuco CRM, Batistoni SST, Lopes A, Cachioni M, Falcão DVS, Neri AL, et al. Mini-Mental State Examination performance in frail, pre-frail, and nonfrail community dwelling older adults in Ermelino Matarazzo, São Paulo, Brazil. Int Psychogeriatr 2012; 24:1725-31.

Submitted on 14/Aug/2013

Final version resubmitted on $24 / \mathrm{Feb} / 2014$

Approved on 12/Mar/2014 